\title{
Theory of selective excitation of multiple-quantum transitions
}

\author{
W. S. Warren, D. P. Weitekamp, and A. Pines \\ Department of Chemistry and Materials and Molecular Research Division, Lawrence Berkeley Laboratory, \\ University of California, Berkeley, California 94720 \\ (Received 18 April 1980; accepted 12 May 1980) \\ The question of whether a molecule can be made to absorb and emit photons only in groups of $\boldsymbol{n}$ is treated. \\ Pulse sequences are introduced which in effect selectively induce the absorption of only groups of $n$ photons. \\ This causes only $n$-quantum transitions even when many other transitions might be resonant. The technique \\ involves repeated phase shifts of $2 \pi / n$ in the radiation to build up the selected coherences and cancel all other \\ coherences, and is applicable to a wide range of spectroscopic systems. Coherent averaging theory is extended \\ to describe selective sequences and demonstrates that $n$-quantum selectivity is possible to arbitrarily high order \\ in the average Hamiltonian expansion. High-order selectivity requires many phase shifts, however, and for this \\ reason the residual nonselective effects of sequences which are selective to only a finite order are calculated. \\ Selective sequences are applied to the multiple-quantum NMR of oriented molecules, where in combination \\ with time reversal sequences they produce a much more efficient transfer of the population differences into \\ selected coherences than is obtainable by normal wideband pumping. For example, the 10-quantum transition \\ in a 10-spin system can be enhanced by more than four orders of magnitude. Experiments on selective excitation \\ of the 4-quantum transitions in oriented benzene verify the expected enhancement.
}

\section{INTRODUCTION}

It has recently been shown that the Zeeman quantum number selection rule $\Delta m=1$ of conventional Fourier transform NMR can be overcome in a systematic manner by suitably designed pulse sequences, thus permitting the observation of multiple-quantum $(\Delta m=n)$ coherences. ${ }^{1-4}$ The usefulness of observing multiple-quantum transitions can be understood by considering a schematic energy level diagram for a system of $N$ spins $\frac{1}{2}$ without symmetry, shown in Fig. 1. The number of levels for each allowed eigenvalue of $I_{4} \equiv m$ from $m=N / 2$ to $m=-N /$ 2 is $\left({ }_{m+(N / 2)}\right)$. With this distribution, the number of allowed single-quantum transitions increases exponentially with $N$, and in general the single-quantum transitions are only resolvable if there are few spins, or if many of the transitions are either degenerate or forbidden. However, there is only one transition with $\Delta m=N$, corresponding to all $N$ spins flipping together, and at most $2 N$ transitions with $\Delta m=N-1$. In an oriented system such as a liquid crystal, the total spectral width may be many $\mathrm{kHz}$, with each line typically a few $\mathrm{Hz}$ wide. Thus, high multiple-quantum spectra are well resolved even if $N$ is large.

This point is illustrated by Fig. 2, which shows the multiple-quantum spectra of benzene oriented in a liquid crystalline solvent. The six-quantum spectrum has only one line, and the five-quantum spectrum has only two lines because of the high molecular symmetry; the normal single-quantum spectrum has 76 lines. ${ }^{5}$ Unfortunately, the enhanced simplicity of multiple-quantum spectra has to be balanced against a reduced overall intensity. On the average, every symmetry allowed transition is pumped about equally well (independent of $\Delta m$ ) in the standard multiple-quantum experiment. ${ }^{6}$ As a result, total intensities of the high multiple-quantum spectra in these totally nonselective experiments are much weaker than the total intensity of the single-quantum spectrum, and most of the spectral intensity is "wasted" in unresolvable transitions.
Clearly, it would be extremely valuable if we were able to distribute the total spectral intensity between only a few orders of coherence, instead of driving all transitions equally. From the viewpoint of perturbation theory this appears impossible because a multiple-quantum transition occurs only with irradiation which also excites lower quantum transitions, particularly when all

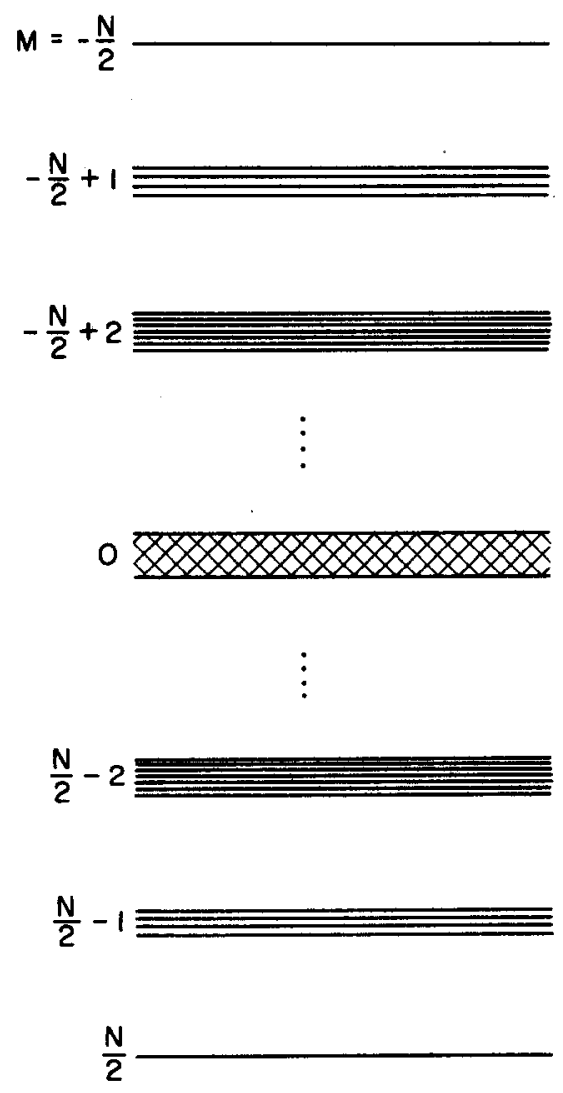

FIG. 1. Energy level diagram for a general system with $N$ spins $\frac{1}{2}$. Each value of $M$ has degeneracy $(N+(N / 2))$, corresponding to a binomial distribution. 


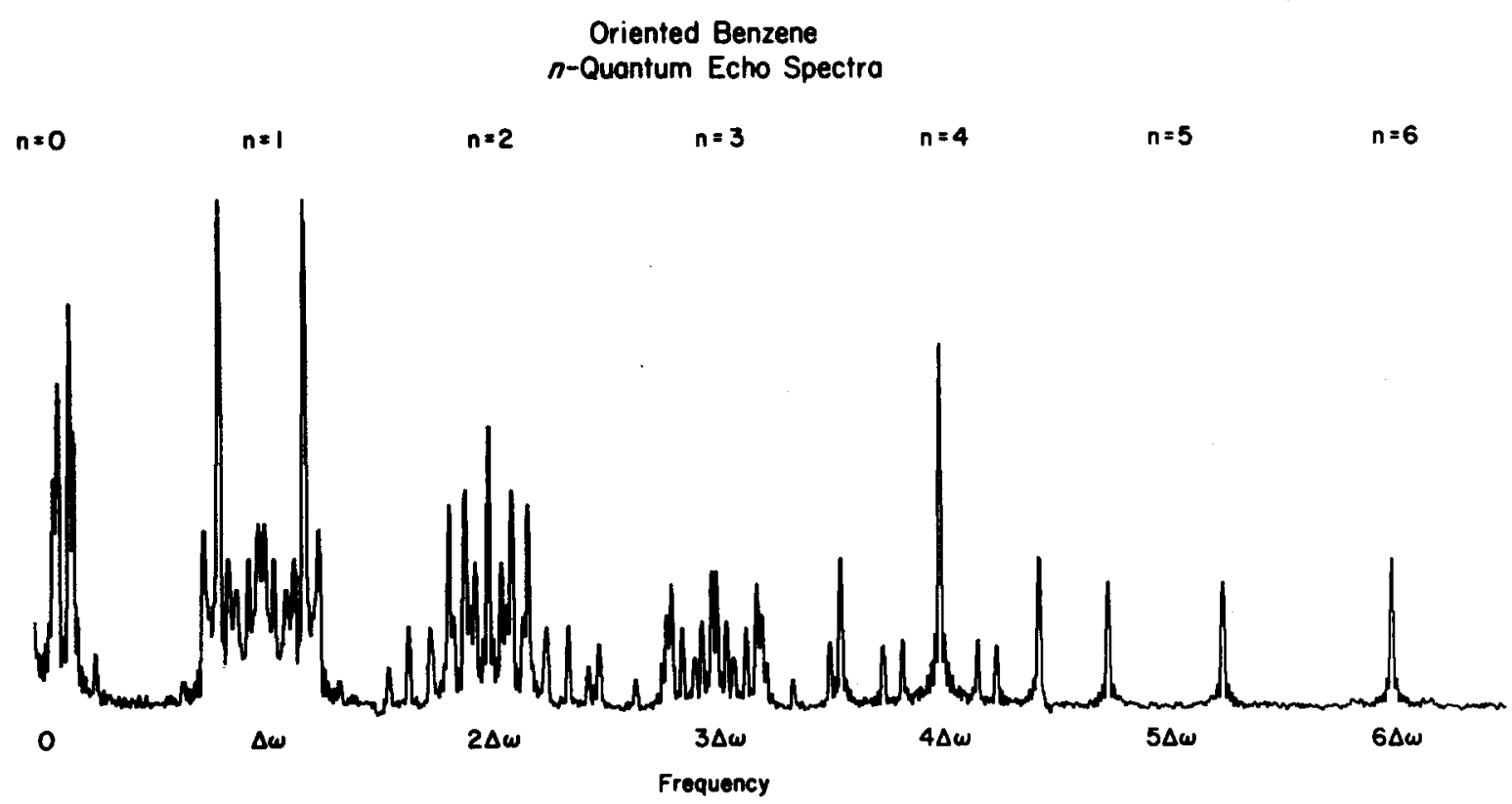

FIG. 2. The multiple-quantum spectra of benzene oriented in a liquid crystal solvent. The number of allowed transitions decreases dramatically as $\Delta M$ increases.

transitions are simultaneously resonant as required by the nearly equal spin energy level spacing. Thus, aside from even-odd selection due to the bilinear form of spin coupling operators, ${ }^{8}$ no general method of selective excitation has been proposed. In this paper and the letter which preceded it, ${ }^{7}$ we demonstrate that selective excitation of multiple-quantum coherences in NMR is theoretically and experimentally possible as illustrated in Fig. 3. We will show that this technique can provide enormous signal enhancement, and that general selective sequences are applicable to a wide range of spectroscopic systems.

\section{GENERAL THEORY OF SELECTIVE SEQUENCES}

\section{A. Review of average Hamiltonian theory}

The effect of any sequence of irradiating pulses and delays on a general system in the absence of relaxation can be represented by a single unitary transformation $U$, called the propagator. Calculating $U$ directly by multiplying together the propagators for each part of the sequence is extremely tedious if many eigenstates are involved. However, this calculation can be avoided for certain pulse sequences by a technique known as average Hamiltonian theory. This technique is thorough-

\section{Oriented Benzene \\ 0,4-Quantum Selective Excitation}

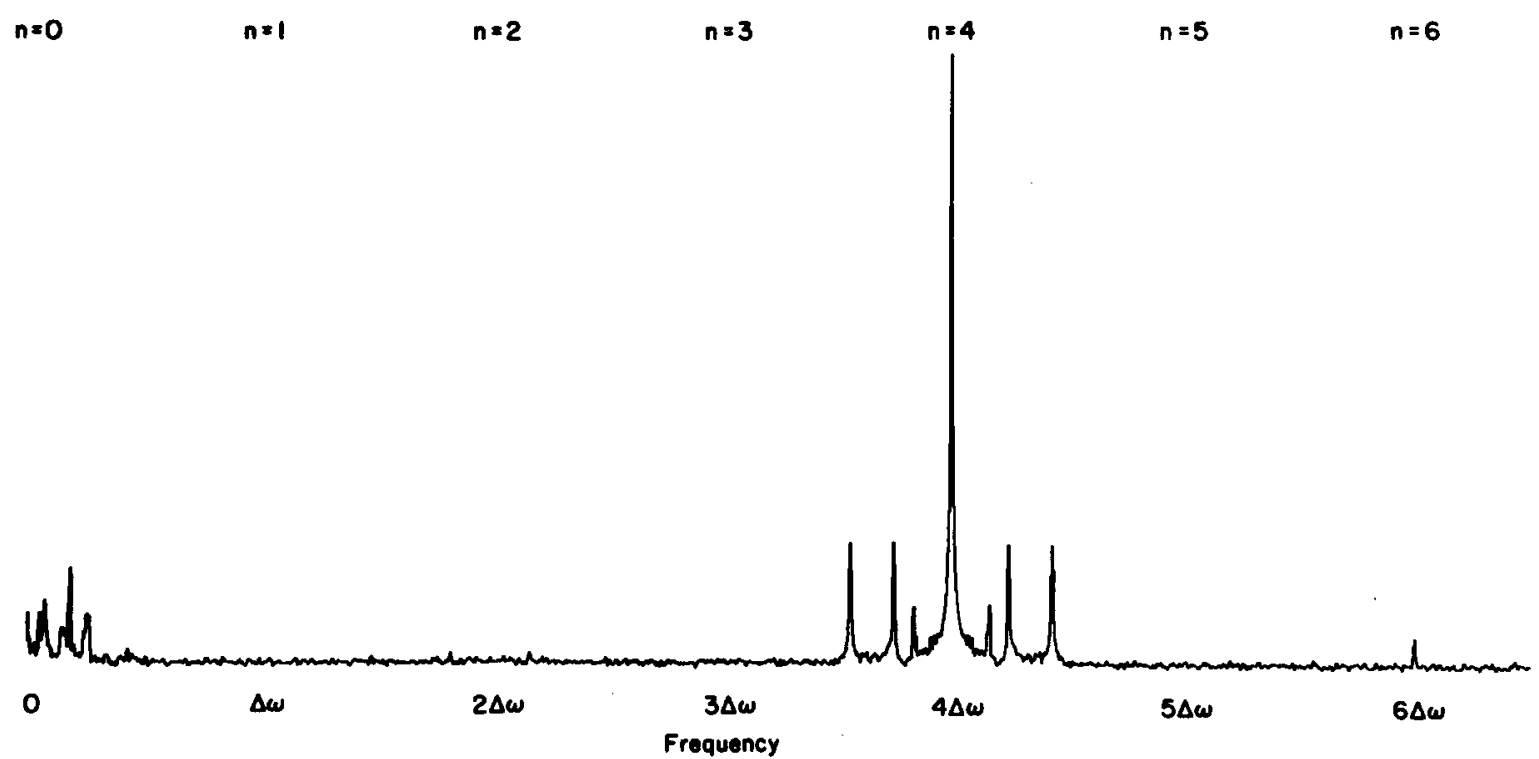

FIG. 3. The multiple-quantum spectra of benzene oriented in a liquid crystal solvent, using selective excitation of zero-quantum and four-quantum transitions. 
ly documented, ${ }^{8,9}$ so we will reproduce in this subsection only a brief summary of previous results which we require for our development. In the next section we will generalize the results to describe sequences which are inherently selective.

The total Hamiltonian of a system is written as $\mathcal{H}(t)$ $=\mathfrak{H}_{\text {Int }}+\mathfrak{H}_{1}(t)$, where $\mathfrak{K}_{1 \mathrm{nt}}$ is the internal Hamiltonian of the system (for example, the interactions between pairs of magnetic dipoles) and $\mathcal{H}_{1}(t)$ is the explicitly time-dependent interaction controlled by the experimenter (for example, the interaction with radiation). $\mathcal{H}_{1}(t)$ is termed cyclic with cycle time $t_{c}$ if $\mathcal{H}_{1}(t)$ and the propagator $U_{1}(t)$ $=T \exp \left[-i \int_{0}^{t} \mathcal{H}_{1}\left(t^{\prime}\right) d t^{\prime}\right]$ ( $T$ is the Dyson time-ordering operator) are periodic, and if $t_{c}$ is the shortest interval that constitutes a period for both $U(t)$ and $\mathcal{H}_{1}(t)$.

$\mathfrak{K}_{\text {in } t}$ is considered to be time independent, so $\mathfrak{H}(t)$ is cyclic if $\mathfrak{K}_{1}(t)$ is cyclic. If $\mathfrak{K}_{1}(t)$ is a pulse sequence made up of an integral number $N$ of cycles, the propagator for the entire sequence is the $N$ th power of the propagator corresponding to one cycle, and therefore only a single cycle need be considered.

The propagator for a single cycle can be shown to be

$$
U=\exp \left(-i \mathcal{K}_{c}\right)=\exp \left[-i\left(\mathcal{H}^{(0)}+\overline{\mathcal{K}}^{(1)}+\cdots+\overline{\mathcal{K}}^{(n)}+\cdots\right) t_{c}\right]
$$

where

$$
\begin{aligned}
& \mathfrak{H}^{(0)}=\frac{1}{t_{c}} \int_{0}^{t_{c}} \tilde{\mathscr{H}}_{1 \mathrm{nt}}(t) d t, \\
& \overline{\mathfrak{K}}^{(1)}=\frac{-i}{2 t_{c}} \int_{0}^{t_{c}} d t_{2} \int_{0}^{t_{2}} d t_{1}\left[\tilde{\mathfrak{H}}_{1 \mathrm{nt}}\left(t_{2}\right), \tilde{\mathfrak{K}}_{\mathbf{i n t}}\left(t_{1}\right)\right], \\
& \overline{\mathcal{K}}^{(2)}=-\frac{1}{6 t_{c}} \int_{0}^{t_{c}} d t_{3} \int_{0}^{t_{3}} d t_{2} \int_{0}^{t_{2}} d t_{1}\left\{\left[\left(\tilde{\mathcal{F}}_{\mathrm{int}}\left(t_{3}\right),\right.\right.\right.
\end{aligned}
$$

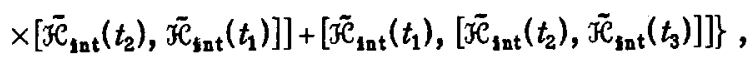

and

$$
\tilde{\mathcal{H}}_{1 \mathrm{nt}}(t)=U_{1}^{-1}(t) \mathcal{H}_{\mathrm{int}} U_{1}(t) .
$$

This is merely a Magnus expansion ${ }^{10}$ of the propagator in powers of the cycle time. The average Hamiltonian expansion is a perturbation expansion in powers of a smallness parameter $t_{c}$ that has a physical meaning; $t_{c}$ and $\mathcal{K}_{\mathrm{int}}(t)$ are simultaneously varied by lengthening the sequence. For this reason, $\overline{\mathcal{H}}^{(i)}$ is termed a correction term of order $i$ and is proportional to $\left(t_{c}\right)^{t} . \overline{\mathcal{H}}^{(0)}$ is the zero-order or average Hamiltonian, and $\mathcal{H}$ is the effective Hamiltonian. The advantage of Eq. (1) is that a complex time dependent process has been expressed by a time independent Hamiltonian.

Pulse sequences are usually designed so that $\overline{\mathfrak{F}}^{(0)}$ has some particular desired property, and then higher-order terms are minimized. For example, line narrowing sequences for solids completely suppress the dipolar or quadrupolar interaction, but not the chemical shift interaction, in this lowest-order term. ${ }^{11}$ If a pulse sequence is symmetric, such that $\tilde{\mathcal{H}}_{\text {int }}(t)=\widetilde{\mathcal{H}}_{1 \mathrm{nt}}\left(t_{c}-t\right), \overline{\mathfrak{H}}^{(1)}$ and all other odd-order correction terms vanish. Symmetric pulse sequences are easy to design, so the major contributions to residual linewidths for simple line narrowing sequences come from $\overline{\mathfrak{H}}^{(2)}$ and from pulse se- quence imperfections (inhomogeneity, timing errors, and the like). A very powerful method of eliminating these terms involves alternating between two or more different cycles (called subcycles) to form a new, larger cycle which has smaller higher-order terms. Under certain conditions, some of the higher-order terms for the entire cycle are simply equal to the sum of the corresponding terms for the subcycles; such terms are said to decouple. ${ }^{12}$ Decoupled pulse cycles for line narrowing have been produced that have $\overline{\mathcal{H}}^{(2)}=0$ for the dipolar Hamiltonian, and have small error terms. ${ }^{12}$

Higher-order terms are usually difficult to calculate, but for line narrowing sequences their size (and therefore their contribution to residual line widths) can be estimated. If $\overline{\mathcal{H}}^{(0)}=\overline{\mathscr{H}}^{(1)}=\overline{\mathcal{H}}^{(2)}=\ldots=\overline{\mathscr{H}}^{(n-1)}=0$, then $\overline{\mathscr{K}}^{(k)}=\overline{\bar{H}}^{(k)}$, where $\overline{\overline{\mathcal{H}}^{(k)}}$ is defined as

$$
\begin{aligned}
& \overrightarrow{\mathcal{H}}^{(k)}= \frac{(-i)^{k}}{t_{c}} \int_{0}^{t_{c}} d t_{k+1} \int_{0}^{t_{k+1}} d t_{k} \cdots \int_{0}^{t_{2}} d t_{1} \tilde{\mathcal{K}}_{1 \mathrm{nt}}\left(t_{k+1}\right) \\
& \times \tilde{\mathfrak{K}}_{1 \mathrm{nt}}\left(t_{k}\right) \ldots, \\
& k=n, \quad n+1, \ldots, 2 n .
\end{aligned}
$$

Reference 9 (a) contains a weaker version of this theorem, which requires $\overline{\mathcal{K}}^{(j)}=0$ for all $j<n$ for $\overline{\mathcal{F}}^{(n)}=\overline{\mathcal{H}}^{(n)}$, but inspection of their proof ${ }^{13}$ leads to the immediate conclusion that $\overline{\mathfrak{K}}^{(j)}=0$ for all $j<(n-1) / 2$ is sufficient for $\overline{\mathcal{K}}^{(n)}=\overline{\bar{K}}^{(n)}$.

The volume of integration is $\left(t_{c}\right)^{n+1}$, so $\overline{\mathcal{F}}^{(n)}$ can be easily estimated in terms of $\mathfrak{K}_{\text {int }}$. To do this estimation we need to use the concept of the norm of a matrix. We will define the norm of an arbitrary $N_{T} \times N_{T}$ matrix $A$ as

$$
\|A\|=\left[\frac{1}{N_{T}} \operatorname{Tr}\left(A A^{\dagger}\right)\right]^{1 / 2} .
$$

$\|A\|$ is invariant under unitary transformations, so if $A$ is Hermitian, $\|A\|$ is the root-mean square eigenvalue of $A$, called $M_{2}^{1 / 2}(A)$. Other convenient properties that are easily proven are as follows:

(1) If $A$ and $B$ are Hermitian, $\|A B\|=\|B A\| \leq N_{T}\|A\|\|B\|$.

(2) $\|I\|=1$, where $I$ is the identity matrix.

(3) If $A$ is Hermitian, $\| A^{n_{\|}}$is the square root of the (2n)th moment of the distribution of the eigenvalues, called $M_{2 n}^{1 / 2}(A)$. Since $M_{2 n}^{1 / 2}(A) \geq\left[M_{2}^{1 / 2}(A)\right]^{\mathrm{n}}$ for any distribution, $\left\|A^{n}\right\| \geq(\|A\|)^{n}$.

(4) If $A$ and $B$ are similar Hermitian matrices, such that $A=U B U^{\dagger},\|A B\| \leq\left\|A^{2}\right\|=\left\|B^{2}\right\|$.

(5) However, if $A$ and $B$ are two different matrices, with nothing else known about either matrix, then $(A B)_{m n}=\sum_{i} A_{m i} B_{i n}$ is the sum of $N_{T}$ numbers, which we expect will add randomly. Then $\left\langle\left|(A B)_{m n}\right|^{2}\right\rangle$ $\sim N_{T}\left\langle\left|A_{m i} B_{i n}\right|^{2}\right\rangle \sim N_{T}\left(\left\langle\left|A_{m i}\right|^{2}\right\rangle\left\langle\left|B_{i n}\right|^{2}\right\rangle\right)^{1 / 2}$, and this implies $\|A B\| \sim\|A\|\|B\|$.

Properties (2) and (4) imply that $\| \tilde{\mathcal{H}}_{\text {int }}\left(t_{n+1}\right)$ $\times \tilde{\mathcal{H}}_{\text {int }}\left(t_{n}\right) \cdots \tilde{\mathcal{H}}_{1 n t}\left(t_{1}\right)\|\leq\|\left[\mathcal{H}_{\text {int }}\left(t_{1}\right)\right]^{n+1} \|$. For many systems the eigenvalues of $\mathfrak{H}_{1 n t}$ have roughly a Gaussian distribution, and in this case 


$$
\begin{aligned}
M_{2 n}^{1 / 2} & =[1 \cdot 3 \cdot 5 \ldots(2 n-1)]^{1 / 2}\left(M_{2}^{1 / 2}\right)^{n} \\
& =\left[(2 n) ! /\left(2^{n}\right) n !\right]^{1 / 2}\left(M_{2}^{1 / 2}\right)^{n} .
\end{aligned}
$$

Thus,

$$
\left\|\overline{\mathcal{H}}^{(n)} t_{e}\right\| \leq\left(\left\|\mathcal{H C}_{9 \mathrm{nt}} t_{a}\right\|^{n+1}\right)\left\{(2 n) ! / 2^{n} n ![(n+1) !]^{2}\right\}^{1 / 2} .
$$

In fact, if the cycle contains many pulses so that $\tilde{\mathcal{F}}_{\mathrm{fnt}}(t)$ varies rapidly, we expect that $\left\|\tilde{\mathcal{H}}_{1 n t}\left(t_{n+1}\right), \tilde{H}_{i n t}\left(t_{n}\right) \ldots \tilde{H}_{\text {int }}\left(t_{1}\right)\right\|$ $\sim\left\|\mathcal{K}_{\mathrm{tnt}}\right\|^{n+1}$. Thus, $\left\|\mathcal{K}^{(n)} t_{c}\right\| \sim\left\|\mathcal{K}_{1 \mathrm{nt}} t_{c}\right\|^{n+1} /(n+1) !$, and for those terms for which $\overline{\mathcal{K}}^{(m+1)}=\overline{\mathcal{K}}^{(m+1)},\left\|\overline{\mathcal{H}}^{(n+1)}\right\| / 1 \mid \overline{\mathcal{K}}^{(n)} \|$ $\sim\left\|\mathfrak{F C}_{\mathrm{fnt}} t_{\mathrm{c}}\right\| /(n+1)$. For higher-order terms expressions involving commutators, such as Eqs. (3) and (4), are required.

All of the results presented so far are applicable to any cyclic pulse sequence. We will now extend average Hamiltonian theory, in order to create pulse sequences which selectively excite only a few transitions.

\section{B. Extension of average Hamiltonian theory to selective sequences}

\section{Definitions}

We term an operator nk-quantum selective if it can be completely decomposed into irreducible tensors $T_{n k}^{j}$, with $k$ allowed to have any integral value including 0 . If only $k= \pm 1$ is required, the operator is termed $n$-quantum selective. If tensor components that are not integral multiples of $n$ are required, the operator is nonselective. Any nonselective operator can be decomposed into a $n k$-quantum selective operator and a remainder which we call non-nk-quantum selective (abbreviated nns). From the definition of tensor operators, the product or sum of two $n k$-quantum operators is also an $n k$-quantum operator. In addition, an operator is $n k$-quantum selective if and only if it is invariant to a rotation of $2 \pi / n$ about the $z$ axis.

We term a cyclic pulse sequence $j$-order $n k$-quantum selective if all the operators $\overrightarrow{\mathcal{H}}^{(i)}(i \leq j)$ in the average Hamiltonian expansion of the propagator are $n k$-quantum selective operators. (For example, if $\overline{\mathfrak{K}}^{(0)}$ is $4 k$-quantum selective but $\overline{\mathcal{H}}^{(1)}$ is not, the sequence is zero-order $4 k$-quantum selective.) An equivalent definition is that all terms in the propagator proportional to $\left(t_{c}\right)^{i+1}(i \leq j)$ are $n k$-quantum selective. If the initial density matrix has no coherences, the final density matrix will contain only $n k$-quantum selective operators, up to terms proportional to $\left(t_{c}\right)^{j+1}$.

The physical meaning of $n k$ quantum operators depends on the system being considered. If the axis of propagation of the radiation is chosen as the $z$ axis, an $n k$-quantum operator causes a net absorption or emission of a multiple of $n$ photons, and changes the $z$ component of the angular momentum of the applied field by some multiple of $n \hbar$. If the $z$ component of angular momentum is a good quantum number for the system (as it is, for example, in NMR at normal magnetic field strengths), conservation of angular momentum implies that the system can develop coherences only between states for which this quantum number differs by a multiple of $n$. If this is not a good quantum number, the selection rules for $n$-quantum transitions are more complicated.

\section{Theorems for selective sequences}

Many of the theorems of average Hamiltonian theory are directly applicable to selective sequences. In addition, we present two new theorems which can be viewed as a generalization of known theorems for line narrowing sequences.

Theorem I: Suppose a cycle (cycle time $t_{c}$ ) consists of $m$ subcycles (cycle times $t_{c 1}, t_{c 2}, \ldots, t_{c m}$ ), each of which is $j$-order $n k$-quantum selective. Then the cycle is also $j$-order $n k$-quantum selective. Furthermore, the non-nk-quantum selective (nns) part of $\overline{\mathfrak{F}}^{(j+1)}$ for the cycle decouples, i.e.,

$$
\left(\overline{\mathcal{C}}^{(j+1)} t_{e}\right)_{\mathrm{sas}}=\sum_{i=1}^{m}\left(\overline{\mathcal{F}}_{i}^{(j+1)} t_{c i}\right)_{\mathrm{nas}} .
$$

Proof: For simplicity of notation we will explicitly prove only the case $m=2$, since repeated application of this theorem with $m=2$ proves the theorem for arbitrary $m$.

Because the propagator for the cycle is equal to the product of the propagators for the two subcycles, we have

$$
\begin{aligned}
& \exp \left[-i\left(\overline{\mathscr{H}}^{(0)}+\overline{\mathcal{H}}^{(1)}+\cdots\right) t_{c}\right]=\exp \left[-i\left(\overline{\mathcal{H}}_{2}^{(0)}+\overline{\mathcal{H}}_{2}^{(1)}+\cdots\right) t_{c 2}\right] \\
& \times \exp \left(-i\left(\overline{\mathcal{K}}_{1}^{(0)}+\overline{\mathfrak{H}}_{1}^{(1)}+\cdots\right) t_{c 1}\right] \text {. }
\end{aligned}
$$

By expanding in powers of $t_{c}, t_{c 1}$, and $t_{c 2}$ and recalling that $\overline{\mathfrak{K}}^{(k)} \sim t_{c}^{k}$ and $\overline{\mathcal{K}}_{f}^{(k)} \sim t_{c j}^{k}$, we find that the term propor tional to $\left(t_{c}\right)^{k+1}$ is

$$
\begin{aligned}
& -i \overline{\mathcal{K}}^{(k)} t_{c}+\frac{1}{2} \sum_{k^{\prime}, K^{\prime}}^{\prime} \overline{\mathcal{H}}^{\left(k^{\prime}\right)} \overline{\mathcal{K}}^{\left(k^{\prime}\right)} t_{c}^{2}+\cdots \\
& =\sum_{k_{1}+k_{2}=k}\left(-i \overline{\mathcal{K}}_{2}^{\left(k_{2}\right)} t_{c 2}+\frac{1}{2} \sum_{k_{2}^{\prime}, k_{2}^{\prime}}^{\prime} \overline{\mathcal{H}}_{2}^{\left(k_{2}\right)} \overline{\mathcal{H}}_{2}^{\left(k_{2}^{\prime}\right)} t_{c 2}^{2}+\cdots\right) \\
& \times\left(-\overline{i \mathcal{C}_{1}^{\left(k_{1}\right)}} t_{c 1}+\frac{1}{2} \sum_{k_{1^{\prime}}, k_{1}^{\prime}}^{\prime} \overline{\mathcal{K}_{1}^{\left(k_{1}^{\prime}\right)}} \overline{\mathcal{K}_{1}^{\left(k_{1}^{\prime}\right)}} t_{c 1}^{2}+\cdots\right), \\
& k^{\prime}+k^{\prime \prime}=k-1 \text {, } \\
& k_{1}^{\prime}+k_{1}^{\prime \prime}=k_{1}-1 \text {, } \\
& k_{2}^{\prime}+k_{2}^{\prime \prime}=k_{2}-1 \text {, }
\end{aligned}
$$

where the terms represented by (...) are products of three or more operators, multiplied by $\left(t_{e}\right)^{3}$ or higher powers of $t_{c} . \overline{3}^{(k)}$ can only appear in the first term on the lhs, and all other terms must have smaller superscripts. By assumption, $\overline{\mathfrak{K}}^{\left(h_{i}\right)}$ is $n k$-quantum selective for all $k_{i} \leq j$. When $k=0$, Eq. (11) simplifies to

$$
i \overline{\mathcal{C}}^{(0)} t_{c}=i\left(\overline{\mathcal{K}}_{1}^{(0)} t_{c 1}+\overline{\mathcal{H}}_{2}^{(0)} t_{c 2}\right)
$$

so $\overline{\mathcal{C}^{(0)}}$ is $n k$-quantum selective if $j \geq 0$. It follows by induction that all the operators $\overline{\mathcal{H}}^{(k)}(k \leq j)$ are $n k$-quantum selective by considering progressively higher powers of $t_{e}$, through $\left(t_{c}\right)^{f+1}$.

The only possible nns term proportional to $\left(t_{c}\right)^{j+2}$ on the lhs is then $\left(-\bar{W}^{(j+1)} t_{c}\right)_{\text {nas }}$ since all other terms involve only lower-order operators which are $n k$-quantum selective. Similarly, the only possible nns term on the 
rhs is $-i\left(\overline{\mathcal{F}}^{(j+1)} t_{c 1}+\overline{\mathcal{H C}}^{(j+1)} t_{c 2}\right)_{\text {nus }}$. By equating these two expressions, Theorem $I$ is proven.

The only property of $n k$-quantum selective operators that was needed to prove Theorem I was closure of this set of operators under addition and multiplication, and similar theorems can be proven for any other set of operators with these two closure properties. In particular, the null set is closed under addition and multiplication. A decoupling theorem for this case (i.e., $\overline{\mathcal{H}}^{(0)}=\overline{\mathcal{H}}^{(1)}=\cdots=\overline{\mathcal{H}}^{(j)}=0$ ) was proven by Burum and Rhim. ${ }^{12}$ Another set which satisfies the required closure properties is the set of $N$-quantum operators in an $N$ spin- $\frac{1}{2}$ NMR system, plus the populations of the two extreme sates; this case will be discussed later.

The size of the first nns term for a $j$-order $n k$-quantum selective sequence can be readily estimated.

Theorem II : If a sequence is $j$-order $n k$-quantum selective, the non- $n k$-quantum selective (nns) part of $\overline{\mathfrak{F}}^{(j+1)}$ can be written as

$$
\begin{aligned}
& \left(\overline{\mathcal{K}}^{(j+1)}\right)_{\mathrm{nns}}=\left[(-i)^{j+1} / t_{c}\right] \\
& \quad \times\left[\int_{0}^{t_{c}} d t_{j+2} \cdots \int_{0}^{t_{2}} d t_{1} \tilde{\mathcal{K}}_{1 \mathrm{nt}}\left(t_{j+2}\right) \cdots \overline{\mathcal{K}}_{\mathrm{int}}\left(t_{1}\right)\right]_{\mathrm{nns}} .
\end{aligned}
$$

Proof: The proof of this theorem is identical to the proof that $\overline{\mathfrak{H}}^{(j+1)}$ has this form if $\overline{\mathcal{K}}^{(j)}=0$ for all $i \leq j$, contained in Ref. 9(a), so we will merely outline it. The most general expression for the term proportional to $\left(t_{c}\right)^{j+2}$ in the propagator is

$$
\begin{aligned}
(-i)^{j+2} & \int_{0}^{t_{c}} d t_{j+2} \int_{0}^{t_{j+2}} d t_{j+1} \cdots \int_{0}^{t_{2}} \\
& \quad \times d t_{1} \tilde{\mathcal{F}}_{\mathrm{int}}\left(t_{n+1}\right) \tilde{\mathcal{F}}_{\mathrm{int}}\left(t_{n}\right) \ldots \tilde{\mathfrak{F}}_{\mathrm{int}}\left(t_{1}\right) .
\end{aligned}
$$

Expanding $U=\exp \left[-i\left(\overline{\mathcal{H}}^{(0)}+\overline{\mathcal{H}}^{(1)}+\cdots+\overline{\mathcal{H}}^{(n)}+\cdots\right) t_{c}\right]$ as in the lhs of Eq. (11), the only possible non-nk-quantum selective term proportional to $\left(t_{c}\right)^{j+2}$ is $\left(-i \overline{\mathcal{K}}^{(j+1)} t_{c}\right)_{\mathrm{nns}}$, which proves the theorem.

It should be noted that $\mathrm{Eq}$. (16) is only valid for the first nns term, while if $\overline{\mathfrak{H}}^{(n)}=0$ for all $n<j$ a similar expression holds for all terms up to $\overline{\mathcal{H}}^{(2 n)}$. The difference is that the lhs of Eq. (11) contains operators such as $\overline{\mathcal{H}}^{\left(k_{1}\right)} \overline{\mathcal{H}}^{\left(k_{2}\right)}$, which vanish if either $\overline{\mathcal{H}}^{\left(k_{1}\right)}$ or $\overline{\mathcal{H}}^{\left(k_{2}\right)}$ vanishes, but which are generally $n k$-quantum selective only if both $\overline{\mathcal{H}}^{\left(k_{1}\right)}$ and $\overline{\mathfrak{K}}^{\left(k_{2}\right)}$ are $n k$-quantum selective.

\section{Design of selective sequences}

\section{Zero-order selective sequences}

Starting from any cyclic sequence of pulses and delays, we can produce a sequence which is zero-order $n k$-quantum selective, using a technique which we call phase cycling. This is illustrated in Fig. 4(a). We assume that the cyclic sequence has a duration $\Delta \tau_{p}$ (which we call a subcycle), an effective Hamiltonian $\mathfrak{H C}_{0}=\overline{\mathfrak{F}}_{0}^{(0)}$ $+\overline{\mathcal{H}}_{0}^{(1)}+\cdots+\overline{\mathcal{H}}_{0}^{(n)}+\cdots$, and a propagator $U_{0}$ $=\exp \left(-i \zeta_{0} \Delta \tau_{p}\right)$. At the end of the interval $\Delta \tau_{p}$, the se quence is repeated with all radiation phase shifted by $\phi=2 \pi / n$ about the $z$ axis, giving a new effective Hamiltonian $\mathfrak{H}_{\phi}$ and propagator $U_{\phi} . \mathcal{H}_{\phi}$ is related to $\mathfrak{K}_{0}$ by a (a)

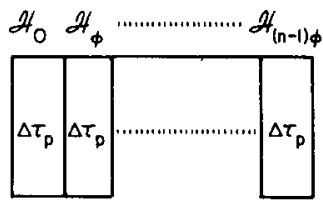

(b)

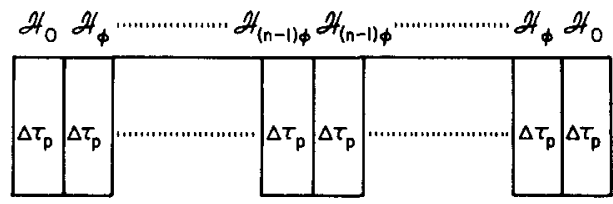

FIG. 4. (a) Phase cycling can be used to create $n k$-quantum selective sequences, using phase shifts of $\phi=2 \pi / n$. The cycle of $n$ subcycles is more selective by one order in the average Hamiltonian theory expansion. (b) The cycle of $2 n$ subcycles formed by phase cycling and symmetrization is more selective by two orders.

rotation of $-\phi$ about the $z$ axis:

$$
\begin{aligned}
& \mathcal{H}_{\phi}=\exp \left(i \phi I_{z}\right) \mathfrak{H}_{0} \exp \left(-i \phi I_{z}\right), \\
& \left(\mathcal{H}_{\phi}\right)_{i j}=\left(\mathcal{H}_{0}\right)_{i j} \exp \left[i \phi\left(m_{i}-m_{j}\right)\right],
\end{aligned}
$$

and $U_{\phi}$ is related to $U_{0}$ in exactly the same manner. This phase shift is repeated $n$ times, creating a cycle with cycle time $t_{c}=n \Delta \tau_{p}$. We would like to calculate $\overline{\mathfrak{H}}^{(j)}$ for the cycle. Since $t_{c}$ is proportional to $\Delta \tau_{p}$ it is clear that $\overline{\mathcal{H}}_{0}^{(j)}$ and $\overline{\mathcal{H}}^{(j)}$ scale in exactly the same manner when $t_{c}$ is changed, and by equating terms proportional to $t_{c}$ with those proportional to $\Delta \tau_{p}$, we find

$$
\overline{\mathcal{H}}^{(0)}=\frac{1}{n} \sum_{i=0}^{n-1} \overline{\mathcal{H}}_{l \phi}^{(0)}=\frac{1}{n} \sum_{i=0}^{n} \exp \left(i l \phi I_{\varepsilon}\right) \overline{\mathcal{H}}_{0}^{(0)} \exp \left(-i l \phi I_{z}\right) \text {. }
$$

This sum scales the matrix element $\left(\overline{\mathcal{F}}_{0}^{(0)}\right)_{i j}$ by $(1 / n)$ $\times \sum_{m=0}^{n-1} e^{i 2 r p l / n}$, where $p=m_{i}-m_{j}$; this scaling factor is zero unless $p=n k$. Therefore, $\overline{\mathcal{H}}^{(0)}$ is a pure $n k$-quantum selective operator. Since $\overline{\mathfrak{H}}^{(0)}$ decouples, any other permutation of the subcycles is also acceptable. Higher order terms have some $n k$-quantum selective parts (for example, there is a contribution $\sum_{l=0}^{n-1} \overline{\mathfrak{H}}_{l \Phi}^{(1)}$ to $\left.\overline{\mathfrak{F}}^{(1)}\right)$ but no higher order terms are completely selective. Thus, the sequence obtained by phase cycling is zero-order $n k-$ quantum selective.

If $t_{c}$ can be made arbitrarily small, all the higherorder terms in the average Hamiltonian expansion become unimportant, and a zero-order selective sequence becomes completely selective; of course, the selective term $\left\|\overline{\mathcal{F}}^{(0)} t_{c}\right\|-0$ as $t_{c}-0$, but this can be remedied by repeating the zero-order sequences many times. In general, however, $t_{c}$ cannot be made arbitrarily small, so higher-order selectivity is desirable. One simple way to get a first-order selective sequence is to symmetrize the cycle, as illustrated in Fig. $4(\mathrm{~b}) . \overline{\mathcal{H}}^{(0)}$ is still $n k$-quantum selective, and the symmetrization causes $\overline{\mathcal{F}}^{(j)}$ to vanish for all odd $j$, so the first nonselective term is $\overline{\mathfrak{H}}^{(2)}$.

\section{Sequences selective to arbitrary order}

Suppose that the sequence for $\mathcal{K}_{0}$ in Fig. $4(\mathrm{a})$ is already $j$-order $n k$-quantum selective, instead of being 
nonselective as was assumed earlier. Theorem I proves that the sequence obtained by phase cycling is $(j+1)$ order $n k$-quantum selective, because $\left(\overline{\mathscr{F}}^{(j+1)}\right)_{\text {nas }}$ decouples:

$$
\begin{aligned}
\left(\overline{\mathcal{H}}^{(j+1)}\right)_{\mathrm{nns}} & =\sum_{i=0}^{n-1}\left(\overline{\mathcal{H}}_{l \phi}^{(j+1)}\right)_{\mathrm{nns}} \\
& =\sum_{i=0}^{n-1}\left[\exp \left(i l \phi I_{z}\right) \overline{\mathcal{H}}_{0}^{(j+1)} \exp \left(-i l \phi I_{z}\right)\right]_{\mathrm{nns}}=0 .
\end{aligned}
$$

Therefore, starting from a nonselective $\mathfrak{H}_{0},(j+1)$ phase cyclings produce a sequence that is $j$-order $n k$ quantum selective, requiring $n^{(j+1)}$ subcycles; each block of $n$ subcycles is zero-order $n k$-quantum selective, each block of $n^{2}$ subcycles is first-order $n k$-quantum selective, and so forth. For example, a first-order $4 k$-quantum selective sequence may be constructed from $4^{2}=16$ subcycles, and the phases of the subcycles can be written schematically $(0: \phi=0,1: \phi=\pi / 2,2: \phi=\pi, 3: \phi=3 \pi / 2)$ as (0123)(1230)(2301)(3012). Each group in parentheses is a zero-order $n k$-quantum selective sequence, and is phase shifted by $\pi / 2$ to produce the next group.

In the absence of relaxation, there is no limit to the number of times phase cycling can be applied, and there fore sequences which are selective to arbitrarily high order can be designed. In any real system, only a limited number of subcycles could be completed before relaxation effects make the average Hamiltonian calculation invalid. One way to reduce the number of subcycles required to achieve a given order of selectivity is to combine phase cycling and symmetrization, as in Fig. 4 (b). The sequence is first-order $n k$-quantum selective even if $\mathcal{K}_{0}$ is nonselective. If $\mathcal{H}_{0}$ is already $j$-order $n k$ quantum selective ( $j$ odd), the phase cycling and symmetrization requires $2 n$ subcycles to make a $(j+2)$-order $n k$-quantum selective sequence, instead of the $n^{2}$ subcycles required for two phase cyclings. Thus, a $(2 j+1)$-order $n k$-quantum selective sequence requires $(2 n)^{j+1}$ subcycles $[(j+1)$ phase cyclings and $(j+1)$ symmetrizations] and a $(2 j)$-order $n k$-quantum selective sequence requires $n(2 n)^{j}$ subcycles $[(j+1)$ phase cyclings and $j$ symmetrizations]. For example, a third-order $4 k-$ quantum selective sequence requires $(2 n)^{2}=64$ subcycles, and the relative phases can be written schematically as (0123)(3210)(1230)(0321)(2301)(1032)(3012)(2103)(3012) (2103) (2301) (1032) (1230) (0321) (0123) (3210).

\section{APPLICATION OF SELECTIVE SEQUENCES TO MULTIPLE-QUANTUM NMR}

\section{A. Motivation}

In this section the general principles of selective excitation are elaborated for the particular case of a system of directly dipole coupled nuclear spins. The analysis of the spectral line positions of such a system supplies structural information on the lengths and orientations of internuclear vectors and analysis of the relaxation times provides information on molecular motion. Neither analysis can be completed unless the spectrum contains resolved assignable lines. The high resolution and simplicity of the high multiple-quantum spectra make them preferable or indispensable for these analyses. It

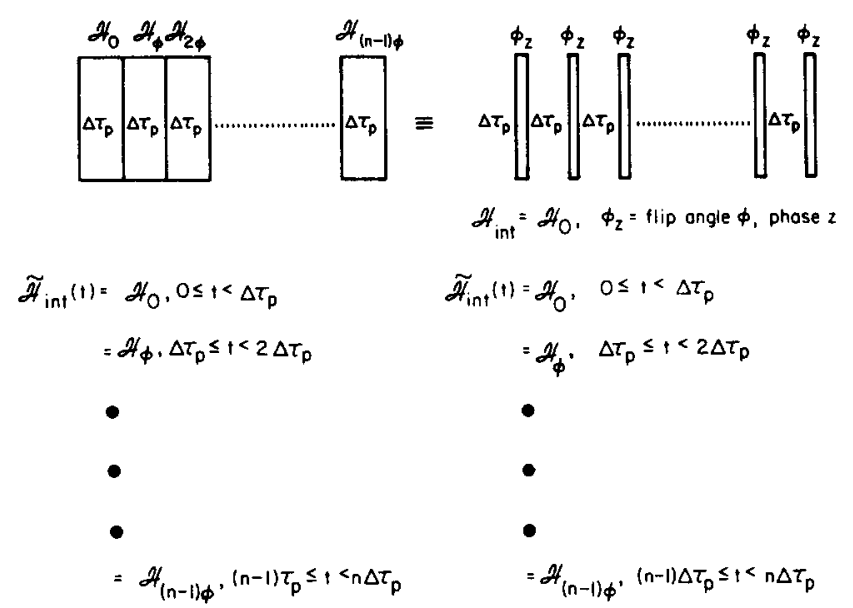

FIG. 5. The analogy between a phase cycled sequence consisting of subcycles with effective Hamiltonians $\mathcal{H}_{\phi}$ and a pulse sequence on a hypothetical system with $\mathcal{H}_{\operatorname{lnt}}=\mathcal{H C}_{0}$. Average Hamiltonian theory can be applied to the pulse sequence.

will be shown how the use of selective sequences promises to overcome the difficulty of small signal intensity, thus making these analyses possible in large spin systems.

The Hamiltonian for the $N$ spins $\frac{1}{2}$ of an oriented molecule in a large magnetic field can be written in the rotating frame (in units of $\hbar=1$ ) as

$$
\mathfrak{H}_{\text {int }}=\mathcal{K}_{\Delta \omega}+\mathcal{H}_{D, x s}+\mathcal{H}_{r f} \text {. }
$$

$\mathcal{H}_{\Delta \omega}=\Delta \omega I_{k}$ is the resonance offset term, $\mathcal{H}_{D_{, k x}}=\sum_{D j} a_{i j}$ $\times\left(3 I_{z i} I_{a j}-I_{i} \cdot I_{j}\right)$ is the secular portion of the dipolar Hamiltonian, and $\mathfrak{C}_{r}$ is the time-dependent interaction between the molecules and applied radiation. Other interactions such as chemical shifts or scalar spin-spin couplings may also be accounted for, but we will not consider them here.

The task we set out to accomplish in this section is to construct a rapidly convergent effective Hamiltonian for an excitation cycle which will contain, in its leading terms, operators $T_{n k}^{j}$ with rank $j$ up to the maximum value $j=N$ and components $n k \leq j$. Such a Hamiltonian will create high quantum coherences selectively even if it acts only for short times such that $\left\|\mathfrak{H C} t_{c}\right\| \ll 1$. The theoretical tool to be used is a nested series of average Hamiltonian expansions with each successive expansion involving a longer cycle time. This approach is exact when the effective Hamiltonian of each of the preceding expansions is used as the starting point for the next expansion. In practice, one designs sequences with rapidly convergent expansions and proceeds by using only the first few terms as an approximation to the effective Hamiltonian. Precedents for this procedure exist. It is implicitly used whenever a rotating frame Hamiltonian is used as a starting point for an average Hamiltonian expansion. "14 Another example is the "second averaging" procedure used to explain off-resonance effects in multiple pulse line narrowing experiments. ${ }^{15}$

The expansions which were the subject of the previous section were the final expansions, in which the effective Hamiltonians $\mathfrak{H}_{\phi}$ for the subcycles were assumed to be known. Figure 5 shows schematically how the phase 
(a)

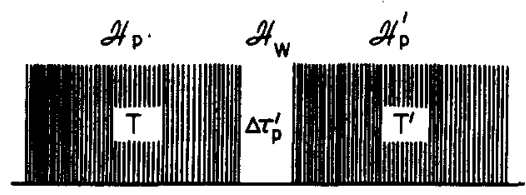

(b)

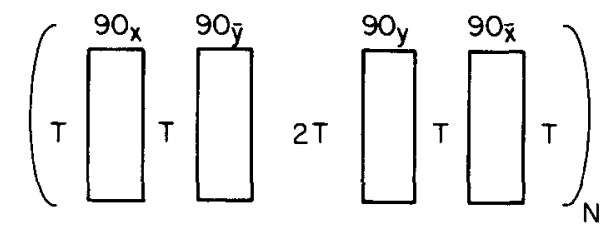

FIG. 6. Possible pulse sequences for the subcycles in Fig. 4. In Fig. 6(a), time reversal sequences generate $\mathcal{K}_{p}^{\prime} T^{\prime}=-\mathcal{K}_{p} T$, so that $\left\|\mathcal{F}_{0}\right\| \ll\left\|\mathcal{K}_{D_{\text {, Aq }}}\right\|$, but $\mathfrak{H}_{0}$ contains multiple-quantum coherences. In Fig. 6(b), a WAHUHA sequence with a long cycle time has the same effect.

shifts of the cycle may be viewed as a series of $z$ pulses on a system with an otherwise time independent Hamiltonian $\mathfrak{H}_{\phi>0}$. In the next section the subcycles are treated.

\section{B. The design of effective subcycles}

If a subcycle can be designed which has high quantum operators in the leading terms of its effective Hamiltonian $\mathcal{H}_{\phi}$, then the cycle need only be selective to a low order since high quantum operators will appear in its leading terms. Since $\mathcal{K}_{i n t}$ contains only first and second rank operators, no simple rotation of $\mathfrak{H}_{\mathrm{int}}$ can contain high quantum operators. It is only through the action of the couplings between spins that such operators can appear in the evolution, so it appears desirable that the subcycle last at least long enough that $\left\|\mathcal{H}_{D, e x} \Delta \tau_{p}\right\|$ $\sim 1$. An apparent dilemma arises here though, for if the cycle consists of many subcycles, then $\left\|\mathcal{H}_{D, k z} t_{c}\right\|>1$ and convergence of the effective Hamiltonian expansion becomes doubtful. The solution to this problem is to ar range the internal structure of the subcycles so that $\left\|\mathcal{H}_{D, \& \text { Ex }} \Delta \tau_{p}\right\| \sim 1$ but $\left\|\mathcal{H}_{\phi} \Delta \tau_{p}\right\| \ll 1$. Several approaches will be discussed. One general approach to subcycle design uses the method of time reversal ${ }^{16}$ and is illustrated in Fig. 6(a). $\mathcal{H}_{p}$ and $\mathcal{H}_{p}^{\prime}$ are approximations to the effective Hamiltonians for the periods $T$ and $T^{\prime}$, respectively. Before examining particular sequences for the periods $T, T^{\prime}$, and $\Delta \tau_{p}^{\prime}$, we indicate how time reversal leads to the desired conditions that $\mathcal{H}_{\phi}$ contains high quantum operators and that $\left\|\mathcal{K}_{\phi} \Delta \tau_{p}\right\| \ll 1$.

Pulse sequences can be designed with $\overline{\mathcal{K}}_{D}^{(0)}=\frac{1}{2}\left(\mathcal{H}_{D, x x}\right.$ $\left.+\mathfrak{H}_{D, y y}\right)=-\frac{1}{2} \mathfrak{H}_{D, \& z}$, and the effect of such sequences is to make the spin system appear to evolve backwards in time. ${ }^{16}$ If such a pulse sequence is applied for a time $2 T$ and then turned off, the initial condition will return after a time $T$. If $\left\|\mathcal{K}_{D, \& \&} T\right\| \gtrsim 1$, both the forward time and reversed time propagators can contain irreducible tensor operators of arbitrarily high rank, but will commute with $I_{x}$. Similarly, pulse sequences can be designed with $\overline{\mathfrak{K}}_{D}^{(0)}=\frac{1}{2}\left(\mathcal{H C}_{D, y y}+\mathcal{H}_{D, z s}\right)=-\frac{1}{2} \mathcal{H}_{D_{0} x x}$. In this case, the propagator obtained from a sequence with $\left\|\mathcal{K}_{D, x x} T\right\| \gtrsim 1$ will contain irreducible tensor operators of arbitrarily high rank, but will not commute with $I_{z}$. Such a propagator can generate multiple-quantum coherences and can be viewed as a multiple-quantum rotation (as opposed to the rotation produced by a single strong pulse, which will only generate single-quantum coherences from a density matrix proportional to $I_{z}$ ).

The propagator for the subcycle of Fig. $6(\mathrm{a})$ is $U\left(\Delta \tau_{p}\right)$ $=\exp \left(-i \mathcal{H}_{p}^{\prime} T^{\prime}\right) \exp \left(-i \mathcal{K}_{w} \Delta \tau_{p}^{\prime}\right) \exp \left(-i \mathcal{F}_{p} T\right)$. Time reversal techniques may be used to arrange that $\mathcal{K}_{p}^{\prime} T^{\prime}$ $=-\mathcal{H}_{p} T$. When this condition holds, the periods $T$ and $T^{\prime}$ may be viewed as a complementary pair of multiplequantum rotations which sandwich the period $\Delta \tau_{p}^{\prime}$. Together they form a cycle and the average Hamiltonian for this cycle is

$$
\overline{\mathcal{H}}_{\phi}^{(0)}=\frac{1}{\Delta \tau_{p}} \int_{0}^{\Delta T_{p}} \tilde{\mathcal{H}}(t) d t=\frac{\Delta \tau_{p}^{\prime}}{\Delta \tau_{p}} e^{i x_{p} T_{\mathcal{K}_{w}}} e^{-i \mathcal{X}_{p} T} .
$$

If $\mathcal{H}_{p}$ is nonsecular, bilinear, and does not commute with $\mathfrak{H}_{w}$, then $\overline{\mathcal{H}}_{\bullet}^{(0)}$ will contain high multiple-quantum operators when $\left\|\mathcal{H}_{p} T\right\| \gtrsim 1$. Since the exponential operators constitute a unitary transformation, the norm of $\mathcal{K}_{w}$ is conserved and $\left\|\overline{\mathcal{F}}_{\phi}^{(0)}\right\|=\left(\Delta \tau_{p}^{\prime} / \Delta \tau_{p}\right)\left\|\mathcal{H}_{w}\right\|$. The desired effect of reducing the norm of the subcycle Hamiltonian is achieved when $\Delta \tau_{p}^{\prime} \ll \Delta \tau_{p}$. Since $\left\|\overline{\mathcal{H}^{(0)}} \Delta \tau_{p}\right\|$ $=\left\|\mathcal{H}_{w} \Delta \tau_{p}^{\prime}\right\|$, the small interval $\Delta \tau_{p}^{\prime}$ may be thought of as an effective cycle time for the subcycle. This concept is useful in that lengthening the cycle time corresponds to lengthening $\Delta \tau_{p}^{\prime}$ and not $T$ or $T^{\prime}$.

Several choices are possible for $\mathfrak{H}_{p}, \mathfrak{H}_{p}^{\prime}$, and $\mathfrak{H}_{w}$. Let $\mathfrak{H}_{D}=\mathcal{H}_{D, x x}=\sum_{D>f} \omega_{i j}\left(3 I_{x i} I_{x j}-I_{i} \cdot I_{j}\right)$ produced by the sequence $90_{y}-T-90_{\bar{y}}$, where 90 is the pulse flip angle, and the subscript is the $\mathrm{rf}$ phase in the rotating frame; $\mathcal{H}_{p}^{\prime}=-\frac{1}{2} \mathcal{H}_{D, x x}$ produced by a time reversing sequence, such as $\left(\frac{1}{2} \tau-90_{x}-\tau-90_{x}-\tau-90_{\bar{z}}-\tau-90_{\bar{z}}-\tau-90_{\bar{x}}-\tau-90_{\bar{z}}-\tau-\right.$ $\left.90_{x}-\tau-90_{x}-\frac{1}{2} \tau\right)$, repeated enough times to fill a period $T^{\prime}=2 T$; and $\mathcal{K}_{w}=\mathcal{H}_{D, z x}$ using no pulses at all $\left(\mathcal{K}_{w}\right.$ is a "window" in the sequence). The particular time reversing sequence chosen for $\mathcal{H}_{p}^{\prime}$ has $\overline{\mathcal{K}}_{D}^{(0)}=-\frac{1}{2} \mathcal{H}_{D, x x} ;$ it is symmetric, so $\overline{\mathcal{H}}_{D}^{(1)}=0$. Using the notation of Ref. 11 for various error terms from pulse imperfections, we find $\overline{\mathcal{H}}_{0}^{(0)}$ (resonance off set and chemical shift terms) $=0$; $\overline{\mathcal{H}}_{D D}^{(0)}$ (rf inhomogeneity effects) $=0$ (to order $\epsilon$ ); $\overline{\mathcal{H}}_{O D}^{(0)}$ (nonzero pulse width) $=0$; and $\overline{\mathfrak{K}}_{B D}^{(1)}=\overline{\mathfrak{K}}_{\mathscr{C}}^{(1)}=\overline{\mathfrak{K}}_{0}^{(1)}=0$ can be achieved by symmetrization. Neglecting all correction terms, we have

$$
\overline{\mathcal{K}}_{\phi=0}^{(0)} \Delta \tau_{p}=\exp \left(i \mathcal{H}_{x x} T\right)\left(\mathcal{H}_{D, z x} \Delta \tau_{p}^{\prime}\right) \exp \left(-i \mathcal{K}_{x x} T\right),
$$

which conserves the even or odd quantum character of the initial density matrix. This is sometimes conveient; for example, a third order $10 k$-quantum selective sequence requires $4 n^{2}=400$ subcycles, but a third order $5 k$-quantum selective sequence only requires 100 subcycles, and if no odd-quantum coherences are present in $\mathcal{H}_{\phi}$, the two sequences have the same effect.

The sequence $90_{y}-T-90_{y}$ only gives $\mathcal{H}_{p}=\mathfrak{H}_{D_{0} x x}$ if there are no chemical shifts, if $\Delta \omega=0$, if the rf homogeneity and the static homogeneity are perfect, and if the pulse widths are negligible. Thus, even if $\tau$ is kept short in the sequences for $T^{\prime}$, it may be that $\left\|\mathcal{H}_{\phi} \Delta \tau_{p}\right\|$ 
(a)
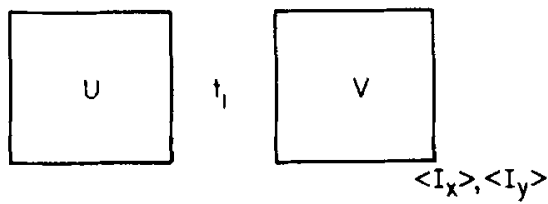

(b)
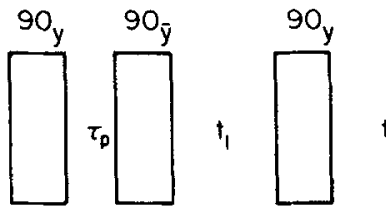

$t_{2}$

$$
<I_{x}>
$$

(c)

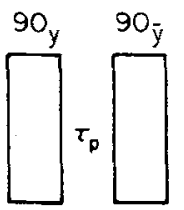

$t_{1}$
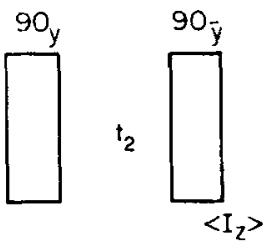

(d)

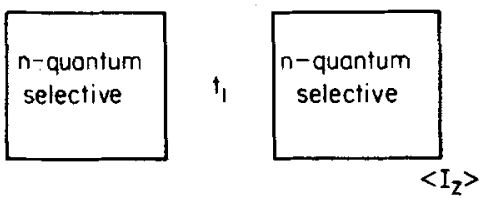

FIG. 7. Pulse sequences for multiple-quantum experiments. (a) is the general case, with preparation propagator $U$ and mixing propagator $V$. (b) is the simplest nonselective multiplequantum experiment. (c) is formally equivalent to (b) and reveals the symmetry between preparation and mixing. (d) is a fully selective experiment, involving selective preparation, mixing, and detection.

$\gg\left\|\mathcal{H}_{w} \Delta \tau_{p}^{\prime}\right\|$ because neglected error terms would enter. One very convenient way to lessen the severity of error terms is to design a sequence with an effective Hamiltonian having only double-quantum terms; then time reversal can be achieved by a phase shift. For example, the sequence $\left(\frac{1}{2} \tau-90_{x}-\tau^{\prime}-90_{x}-\tau-90_{\bar{x}}-\tau^{\prime}-90_{\bar{x}}\right.$ $\left.-\tau-90_{x}-\tau^{\prime}-90_{z}-\tau-90_{x}-\tau^{\prime}-90_{x}-\frac{1}{2} \tau\right)$ has an average Hamiltonian $\overline{\mathfrak{K}}_{D}^{(0)}=\left(\tau^{\prime} / \tau+\tau^{\prime}\right) \mathcal{K}_{D, y y}+\left(\tau / \tau+\tau^{\prime}\right) \mathcal{H}_{D, z x}$ in the limit of $\delta$-function pulses, and if $\tau^{\prime}=2 \tau$, then

$$
\overline{\mathcal{H}}_{D}^{(0)}=\frac{1}{3}\left(2 \mathcal{K}_{D, y y}+\mathcal{H}_{D, E z}\right)=\frac{1}{3}\left(\mathcal{H C}_{D, y y}-\mathcal{H}_{D, x x}\right) \text {. }
$$

This is a pure double-quantum operator, which can be inverted by phase shifting the sequence by $90^{\circ}$. If the pulses are assumed to have a square envelope but a finite width $t_{p}, \overline{\mathscr{H}}_{D}^{(0)}$ is a pure double-quantum operator for $\tau^{\prime}=2 \tau+t_{b}$; other pulse errors may change this relation slightly.

The sequence can be used with $x$ and $\bar{x}$ pulses with enough cycles to create a total length $T$ such that $\left\|J_{\zeta} T\right\|$ $\gtrsim 1$. $\mathfrak{H}_{p}^{\prime}$ is provided by the same sequence, except with $y$ and $\bar{y}$ pulses. Potential sequences for $\mathfrak{K C}_{w}$ include the following:

(1) no pulses, giving $\mathfrak{K}_{w}=\mathfrak{F C}_{D, x \in}$ and even-quantum selection for initial condition $\beta I_{s}$;

(2) $45_{x}-\Delta \tau_{p}^{\prime}-45_{z}$, giving all orders in $\mathcal{K}_{\oplus}$;
(3) the same sequence as $\mathfrak{K}_{p}$, except phase shifted by $45^{\circ}$; clearly $\mathcal{H}_{w}$ is also a pure two-quantum operator, but $\left[\mathfrak{K}_{b}, \mathfrak{H}_{w}\right] \neq 0$, so multiple-quantum coherences still develop; after a brief interval $\Delta T_{p}^{\prime}$, another phase shift of $45^{\circ}$ gives $\mathcal{H}_{b}^{\prime}$.

Experimental work to date $e^{7,17}$ has used $\mathfrak{K}_{w}=\mathfrak{H}_{D, * z}$ and $\mathcal{K}_{p}=\frac{1}{3}\left(\mathcal{K}_{D, y y}-\mathcal{H}_{D, x x}\right)$. Figure 3 is the $4 k$-quantum selective spectrum of oriented benzene obtained with this approach. Previous attempts with $\mathcal{H}_{p}=\mathcal{H}_{D, x x}$ and $\mathcal{H}_{p}^{\prime}$ $=-\frac{1}{2} \mathcal{K}_{D, x x}$, as discussed above, were less successful.

The standard WAHUHA sequence, illustrated in Fig. $6(\mathrm{~b})$, is another possible pulse sequence for $\mathfrak{H}_{\phi}$. If $\mathfrak{H}_{\text {int }}=\mathfrak{K}_{D, E E}$, this sequence gives $\overline{\mathfrak{H}}^{(0)}=\overline{\mathfrak{H}}^{(1)}=0$ (neglecting pulse errors). However, if $\left\|\mathcal{K}_{D, z \ell} T\right\| \gg 1, \mathfrak{H}_{\phi}$ will have strong contributions from ${\overline{\mathrm{K}^{(2)}}}^{(2)} \overline{\mathrm{K}}^{(4)}$, and higher order terms which contain multiple-quantum coherences. For some value of $T$ such that $\left\|\mathfrak{K}_{D, E z} T\right\| \sim 1$, we expect to find $\left\|\mathcal{K}_{\phi}\right\| \ll\left\|\mathcal{K}_{D, \& x}\right\|$, but $\mathcal{K}_{\phi}$ will contain a substantial fraction of multiple-quantum coherences. The sequence is repeated $N$ times, so $\Delta \tau_{p}=6 N T$. When such a subcycle is incorporated into a selective excitation sequence, it will prove useful to think of $T$ as a fixed parameter while $N$ is varied in order to vary the cycle time.

Clearly, any other line narrowing sequence is also a candidate for producing $\mathcal{K}_{\phi}$, but this sequence would probably be the easiest to use because of its relatively large correction terms. A possible advantage over the use of time-reversing sequences is the very low duty cycle, which results because $T$ is much longer than in a normal WAHUHA experiment.

\section{Selective sequences in the multiple-quantum NMR experiment}

Any selective sequence can be incorporated into the general framework of a multiple-quantum experiment, shown in Fig. 7 (a). A large static magnetic field $\mathrm{B}=B_{0} \hat{z}$ is applied to a system of $N$ spin-I nuclei, giving an equilibrium spin density matrix $\rho_{0}=\exp \left(-\beta^{\prime} I_{\alpha}\right) /$

$\operatorname{Tr}\left[\exp \left(-\beta^{\prime} I_{k}\right)\right]$, where $\beta^{\prime}=\gamma B_{0} / k T$; in the high temperature approximation we write the reduced density matrix as $\rho_{0}=(2 I+1)^{-N}\left(-\beta^{\prime} I_{z}\right) \equiv \beta I_{z}$. A pulse sequence (which need not be cyclic) is applied to produce multiple-quantum coherences. This is called the preparation sequence, and we will denote its propagator by $U$. The spins then evolve under $\mathcal{K}_{1 \mathrm{nt}}$ for a time $t_{1}$. In the simplest experiments no pulses are applied during $t_{1}$; how ever, decoupling, spin echoes, or more complicated sequences are possible if suppression of part of $\mathcal{K}_{1 \mathrm{nt}}$ is desired. Because only the operators $I_{x}$ and $I_{y}$ are observable, another pulse sequence (called the mixing sequence) is used to transfer the multiple-quantum coherences that evolved during $t_{1}$ into single-quantum coherences; we will denote the propagator for the mixing sequence by $V$. The oscillating magnetization propor tional to $\left\langle I_{x}\right\rangle$ and $\left\langle I_{y}\right\rangle$ is measured, the experiment is repeated many times with different values of $t_{1}$, and the signal is Fourier transformed with respect to $t_{1}$ to produce the multiple-quantum spectra. In this work it will be assumed that the initial condition is $\beta I_{\alpha}$ and that only the operators corresponding to magnetization at the end 
of the mixing period $V$ are detected. The use of coherently excited initial conditions and the implications of selective excitation for full two dimensional NMR experiments will be discussed elsewhere.

In the simplest experiment, $U$ and $V$ are nonselective as shown in Fig. 7(b). The first two pulses, separated by a time $\tau_{p}$, constitute the preparation sequence. If we further assume that all pulses are strong pulses, so that the internal spin Hamiltonian $\mathfrak{K}_{z}=\Delta \omega I_{z}+\mathfrak{K}_{D, z z}$ can be neglected during the short intervals that the rf field is present, we can write the reduced density matrix at the end of the second pulse as

$$
\begin{aligned}
& \rho=\beta \exp \left(-i I_{y} \pi / 2\right) \exp \left(-i H_{z} \tau_{\rho}\right) \exp \left(i I_{y} \pi / 2\right) I_{z} \exp \left(-i I_{y} \pi / 2\right) \\
& \quad x \exp \left(i H_{z} \tau_{p}\right) \exp \left(i I_{y} \pi / 2\right)=\beta \exp \left(i H_{x} \tau_{\rho}\right) I_{z} \exp \left(i H_{x} \tau_{p}\right),
\end{aligned}
$$

where

$$
\begin{aligned}
& \mathcal{H}_{\alpha}=\Delta \omega I_{\alpha}+\mathcal{H}_{D, \alpha \alpha}(\alpha=x, y, \text { or } z), \\
& \mathcal{H}_{D_{, \alpha \alpha}}=\sum_{i<j} \mathscr{a i j}_{i j}\left(3 I_{\alpha i} I_{\alpha j}-I_{i} \cdot I_{j}\right) \quad(\alpha=x, y, \text { or } z) .
\end{aligned}
$$

Because the dipolar Hamiltonian is bilinear, $\rho$ will have multiple-quantum matrix elements if $\left\|\mathcal{H}_{D, a z} \tau_{D}\right\|$ $\gtrsim 1$, in contrast to excitation by a single pulse which produces only single-quantum coherence. The third pulse and the final delay $t_{2}$ constitute the mixing and detection sequence, and $\left\langle I_{x}\left(t_{2}\right)\right\rangle$ is measured. This pulse sequence, modified to include echoes to remove $\Delta \omega$ and field inhomogeneity from the evolution time $t_{1}$, and phase shifted to separate the different $n$ values, ${ }^{2(c),(d)}$ is the one used for most multiple-quantum experiments.

The dramatic decrease in intensity as $n$ increases and the low intensity of individual transitions mentioned in the Introduction is now easily derived. To simplify the formalism, we note that there is actually a great deal of similarity between the preparation and mixing portions of the pulse sequence, which is hidden by the experimental need to measure $\left\langle I_{x}\right\rangle$ or $\left\langle I_{y}\right\rangle$ even though the initial density matrix is proportional to $I_{k}$.

If we imagine instead that we observe $\left\langle I_{x}\right\rangle$, as in Fig. 7 (c), we have to insert a fourth pulse at the end of $t_{2}$. The sequences in Figs. 7 (b) and 7 (c) would always give exactly the same spectra, but in Fig. 7(c) the symmetry between preparation and mixing is apparent; in fact, if $t_{2}=\tau_{p}$, the propagators are identical:

$$
U=V=\exp \left(-i I_{y} \pi / 2\right) \exp \left(-i H_{z} \tau_{p}\right) \exp \left(i I_{y} \pi / 2\right) .
$$

We can write the observed signal as

$$
\begin{aligned}
\left\langleI _ { k } \left(\tau_{p}, t_{1}, t_{2}\right.\right. & \left.\left.=\tau_{p}\right)\right\rangle=\operatorname{tr}\left(\rho I_{k}\right) \\
& =\beta \operatorname{tr}\left[V \exp \left(-i H_{q} t_{1}\right) U I_{z} U^{\dagger} \exp \left(i H_{k} t_{1}\right) V^{\dagger} I_{z}\right],
\end{aligned}
$$

$$
=\beta \operatorname{tr}\left[\left(U I_{\varepsilon} U^{\dagger}\right) \exp \left(i H_{\varepsilon} t_{1}\right)\left(V^{\dagger} I_{\varepsilon} V\right) \exp \left(-i H_{z} t_{1}\right)\right] \text {, }
$$

$$
=\beta \sum_{m n}\left(U I_{\varepsilon} U^{\dagger}\right)_{m n}\left(U^{\dagger} I_{\varepsilon} U\right)_{n m} e^{i \omega_{m n} t_{1}}
$$

where $\omega_{m n}$ is the energy difference between the states $m$ and $n$. The maximum signal is obtained when $\left|\left(U I_{z} U^{\dagger}\right)_{m n}\right|=\left|\left(U^{\dagger} I_{g} U\right)_{n m}\right|$ for every matrix element, and it can easily be shown that the condition is satisfied if $U$ has the form in Eq. $(29)^{18}$; in fact, for this sequence $\left(U I_{z} U^{\dagger}\right)_{m n}=\left(U^{\dagger} I_{z} U\right)_{n m}$. Therefore, the intensity of the multiple-quantum transition at the frequency $\omega_{m n}$ is equal to $\beta\left|\left(U I_{z} U^{\dagger}\right)_{m n}\right|^{2}$ assuming that all peaks are resolvable. The total intensity of the spectrum, which is divided among all the orders of multiple-quantum transitions, is equal to $\beta \operatorname{Tr}\left[\left(U I_{s} U^{\dagger}\right)^{2}\right]$. However, this quantity is invariant to unitary transformation and is the same as the integrated intensity of the single-quantum spectrum in a conventional one-pulse experiment $\beta \operatorname{Tr}\left(I_{y}^{2}\right)$. Therefore, the total spectral intensity of the nonselective experiment is fixed.

If the multiple-quantum matrix elements are inefficiently excited (for example, if $\left\|\mathfrak{K}_{D, z z} \tau_{p}\right\| \ll 1$ ), $U I_{z} U^{\dagger}$ has large matrix elements along its diagonal. These matrix elements are populations, so they do not evolve, and most of the intensity of the multiple-quantum spectrum is found at $\omega=0$. An efficient wide band nonselective excitation has little intensity at $\omega=0$, and excites all of the possible transitions about equally. Therefore, the average intensity of a single line in a multiple-quantum spectrum is smaller than the average intensity of a single line in an ordinary single pulse experiment, by a ratio (number of single-quantum transitions)/(number of excited multiple-quantum transitions). When there are many spins, the intensity of a single transition becomes extremely small. For example, a system with $N$ spins $\frac{1}{2}$ and no symmetry has $2^{2 N}$ possible distinct matrix elements, so totally nonselective excitation gives a signal for each transition of $2^{-2 N}\left[\beta \operatorname{tr}\left(I_{y}^{2}\right)\right]$.

If only certain orders of multiple-quantum transitions are excited, but the excitation is still efficient (in the sense that the peak at $\omega=0$ is small), the intensity of a single transition grows. For example, if the resonance off set is removed from the excitation and detection periods (by echoes, for example), $H_{x}$ retains only zeroand two-quantum operators, and only even-quantum coherences are excited. ${ }^{6}$ Since roughly half the coherences are even quantum, this process increases the intensity of an average even quantum transition by a factor of 2 . If only a few transitions are excited (by an extremely selective sequence) and the sequence is efficient, the intensity of each transition could be enormous. Suppose that selective excitation is used for both preparation and mixing as in Fig. $7(\mathrm{~d})$, and that $U I_{q} U^{\dagger}$ and $V I_{s} V^{\dagger}$ could be prepared with all the matrix elements zero except for the single coherence with $\Delta m=+N$ and the single coherence with $\Delta m=-N$. In that case, the signal gain relative to the nonselective experiment would be $2^{2 N-1}$. However, the density matrix that results is not related to the initial condition $I_{k}$ by a unitary transformation, and therefore it cannot be produced by any sequence that does not include relaxation.

A more reasonable estimate of the maximum possible gain is obtained by finding the maximum possible value of $\left(U I_{a} U^{\dagger}\right)_{a b}$, where $|a\rangle$ is the single state with $m=+N / 2$ and $|b\rangle$ is the single state with $m=-N / 2$. We have 


$$
\begin{aligned}
\left(U I_{z} U^{\dagger}\right)_{a b} & =\sum_{i} U_{a i}\left(I_{z}\right)_{i i} U_{i b}^{\dagger}, \\
& =\sum_{i} U_{a i}\left(U_{b i}\right) *\left(I_{z}\right)_{i i}, \\
\sum_{i} U_{a i}\left(U_{b i}\right) * & =\delta_{a b} .
\end{aligned}
$$

The maximum can be readily seen to be $U_{a a}=1 / \sqrt{2}$, $U_{a b}=1 / \sqrt{2}, U_{a i}=0, U_{b a}=-1 / \sqrt{2}, U_{b b}=1 / \sqrt{2}$, and $U_{b i}=0$. The phases are not unique. Such a propagator concentrates the matrix elements of $U$ in the states with the largest values of $|m|$. It couples states $|a\rangle$ and $|b\rangle$ only to each other, effectively creating a two-level system. The two-level system has

$$
\begin{aligned}
U=\left(\begin{array}{cc}
1 / \sqrt{2} & 1 / \sqrt{2} \\
-1 / \sqrt{2} & 1 / \sqrt{2}
\end{array}\right) & =\exp \left[i(\pi / 2) I_{y}^{a b}\right], \\
I_{y}^{a b} & =\left(\begin{array}{cc}
0 & -i / 2 \\
i / 2 & 0
\end{array}\right),
\end{aligned}
$$

where $I_{y}^{a b}$ is a fictitious spin- $\frac{1}{2}$ operator for multiplequantum coherence. ${ }^{10}$

Thus, the maximum possible signal is obtained by a selective $90^{\circ}$ pulse, shown schematically in Fig. 8. The signal from this transition is, from Eq. $(32),\left(I_{a}^{2}\right)_{a a}$. The gain when compared to totally nonselective excitation is then

$$
\begin{aligned}
G_{N} & =\left[\beta\left(I_{s}^{2}\right)_{a a}\right] /\left[\beta 2^{-2 N} \operatorname{tr}\left(I_{s}^{2}\right)\right] \\
& =\beta(N / 2)^{2} / \beta 2^{-2 N}\left(N 2^{N} / 4\right)=N 2^{N} .
\end{aligned}
$$

To achieve this gain, we need a sequence that couples the state $m=N / 2$ only to the state $m=-N / 2$. The ef fective Hamiltonian for this sequence should be some linear combination of $I_{y}^{a b}$ and $I_{x}^{a b}$. This sequence would be used to create $U I_{s} U^{\dagger}$ and $V I_{s} V^{\dagger}$. If the effective Hamiltonian has this for $m$ for $m= \pm N / 2$, it can have any form whatsoever for the other levels, and the signal in the $\mathrm{N}$-quantum transition will be unaffected.

Often the $(N-1)$-quantum or $(N-2)$-quantum transitions in an $N$-spin system are more inter esting than the $N$-quantum transition, since the $N$-quantum transition contains no dipolar information. If $(N-1)$-quantum selection on $I_{2}$ is used, the number of transitions increases to $2 \mathrm{~N}$ for a system without symmetry. In ad-
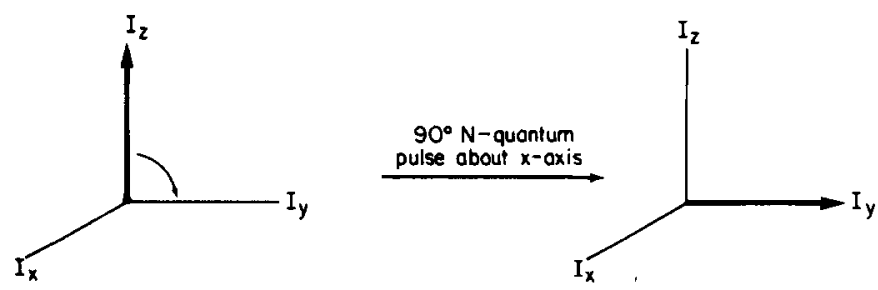

FIG. 8. Schematic illustration of the effect of $N$-quantum selective sequences, in terms of an effective two-level system involving only $m= \pm N / 2$. A selective $90^{\circ}$ pulse transfers the entire population difference between those two states into $N$-quantum coherence, giving a gain relative to nonselective excitation of $N 2^{N}$ (see text).
TABLE I. Enhancement of high multiplequantum transitions, using selective sequences. Sequences which select only $N$ quantum [or only $(N-1)$ quantum] are illustrated in Fig. 4.

\begin{tabular}{rlrc}
\hline \hline$N$ & Symmetry & \multicolumn{1}{c}{$G_{N}$} & \multicolumn{1}{c}{$G_{N-1}$} \\
\hline 2 & None & 8 & 1.14 \\
4 & None & 64 & 6.10 \\
6 & Benzene & 47.5 & 17.2 \\
6 & None & 384 & 32.7 \\
8 & None & 2048 & 154 \\
10 & None & 10240 & 683 \\
12 & None & 49152 & 2922 \\
14 & None & 229376 & 12269 \\
16 & None & 1048576 & 50892 \\
18 & None & 4718592 & 209409 \\
\hline \hline
\end{tabular}

dition, while it is possible to envision a $\pi / 2$ pulse on a two-level system completely depleting the population difference, in a multilevel system it is very unlikely that all population differences can be eliminated simultaneously. Thus, we expect to also produce zero-quantum transitions and populations in the $m= \pm N / 2$ and $m= \pm(N /$ $2-1)$ manifolds, effectively increasing the total number of pumped matrix elements to $2 N^{2}+2$. Now, however, the available fraction of $I_{z}^{2}$ is larger. The result of all of these effects is

$$
\begin{aligned}
G_{N-1}= & \left\{\beta\left[2\left(I_{z}^{2}\right)_{m=N / 2}+2 N\left(I_{z}^{2}\right)_{m=N / 2-1}\right]\right\} / \\
& \times\left(2 N^{2}+2 N+2\right)\left[\beta 2^{-2 N} \operatorname{Tr}\left(I_{z}^{2}\right)\right] \\
= & 2^{N}\left(N^{2}-3 N+4\right) /\left(N^{2}+N+1\right) \approx 2^{N}, \text { for } N \gg 1 .
\end{aligned}
$$

Values of $G_{N}$ and $G_{N-1}$ for systems without symmetry are listed in Table I. If symmetry is included, all gains are reduced, because fewer transitions are allowed and therefore the system is effectively a collection of smaller systems. All of our calculations are still valid, except that the number of density matrix elements excited and the available fraction of $I_{z}^{2}$ should be recalculated using the known symmetry. In general, $N$-quantum and $(N-1)$-quantum transitions must have $A_{1}$ symmetry, since the states with $m= \pm N / 2$ have that symmetry. The relevant energy level diagram is not a binomial distribution but instead is the group of $A_{1}$ states. The calculations are straightforward, and benzene has been included in Table I to illustrate symmetry effects.

These gains become extremely large for large $N$. However, the single $N$-quantum transition contains only a tiny fraction of the total intensity in the nonselective experiment, and therefore we should calculate the total signal available in the $N$-quantum and $(N-1)$-quantum transitions with and without selectivity. This calculations is done in Table II. We assume that the total number of protons in the sample is kept constant as we change $N$, and calculate the signal size as a fraction of the total magnetization of the sample. The signal size still decreases as $N$ increases, but the decrease is much slower in the selective experiment, and Table II indicates that selective excitation should dramatically increase the number 
TABLE II. Intensity of high multiple-quantum transitions, with and without selectivity, relative to total magnetization of the sample.

\begin{tabular}{rllll}
\hline & & \multicolumn{3}{c}{ Intensity (in percent) } \\
\cline { 3 - 5 }$N$ & Symmetry & Nonselective & $x G_{N}$ & $x G_{N-1}$ \\
\hline 6 & Benzene $A_{1}$ & 0.197 & 9.38 & 3.69 \\
6 & None & 0.024 & 9.38 & 0.80 \\
8 & None & $1.5 \times 10^{-3}$ & 3.13 & 0.24 \\
10 & None & $9.5 \times 10^{-5}$ & 0.98 & 0.065 \\
12 & None & $5.9 \times 10^{-6}$ & 0.29 & 0.017 \\
14 & None & $3.7 \times 10^{-7}$ & 0.085 & $4.5 \times 10^{-3}$ \\
16 & None & $2.3 \times 10^{-8}$ & 0.024 & $1.2 \times 10^{-3}$ \\
18 & None & $1.4 \times 10^{-9}$ & 0.0069 & $3.0 \times 10^{-4}$ \\
\hline
\end{tabular}

of molecules which could be studied by multiple-quantum spectroscopy. However, the results in Tables I and II were calculated by assuming that a perfectly selective experiment is possible. Since any actual experiment will deviate from this ideal, we must now consider sequences which are not selective to infinitely high or der, and which can have other errors.

\section{EXTENT OF SELECTIVITY IN NONIDEAL SELECTIVE SEQUENCES}

\section{A. General systems}

As mentioned earlier, for any multiple-pulse sequence one expects $\left\|\overline{\mathfrak{H}}^{(n)}\right\| \lesssim\left\|\mathcal{H}_{i n t}\right\|^{n+1} t_{c}^{n}$. In a selective sequence, $\mathfrak{H}_{\phi}$ is formally equivalent to $\overline{\mathfrak{H}}_{\text {int }}(t)$, as illustrated in Fig. 5. Therefore, as $\left\|\mathcal{H}_{\phi} t_{c}\right\| \rightarrow 0, \mathcal{H}^{(0)}$ becomes the dominant term of $\mathcal{H C}$. The nonselective terms of $U=\exp \left[i \mathcal{H C}\left(N t_{c}\right)\right]$ can be made arbitrarily small in principle by making $t_{c}$ very short, while if $N t_{c}$ is kept constant the selective contribution from $\overline{\mathfrak{H}}^{(0)}$ is unaffected. In practice, the attainable selectivity is limited by several factors.

(1) For technical reasons, $t_{c}$ cannot be made arbitrarily short. For example, if each subcycle requires pulses with specified flip angles, each pulse has a finite width which depends on the strength of the exciting field.

(2) The time required to pump multiphoton coherences is generally dependent on the "anharmonicity" of the energy level spacing. The excitation sequence needs to extend for a period comparable to the inverse of the anharmonic frequencies, which in the last section were the dipolar frequencies. This problem was investigated in depth in the last section; one solution is to construct a subcycle with an effective Hamiltonian $\left\|\mathcal{H}_{\phi}\right\| \ll\left\|\mathcal{K}_{1 \mathrm{nt}}\right\|$, so that $\left\|\mathcal{K}_{\phi} t_{c}\right\|$ can be small even though $\left\|\mathcal{K}_{i n t} t_{c}\right\|$ is not. If this is not possible, the general considerations of the preceding section still hold, but to retain selectivity the subcycles would need to be shorter, and cycles selective to higher order would be needed to obtain high quantum operators.

(3) Because there is a lower limit to the length of a subcycle, the minimum time needed for a $j$-order $n k-$ quantum selective sequence increases rapidly as $j$ increases. However, relaxation mechanisms make the average Hamiltonian calculation invalid if the total length of a sequence is comparable to $T_{2}$, the coherence de- phasing time. Thus, for any system only a finite order of selectivity is possible. Inhomogeneous systems are a special case; excitation designed to compensate for such broadening may allow homogeneous selective excitation.

(4) Timing errors, inaccurate phase shifts, or other failures in control over coherence will reduce the selectivity of any sequence.

In this subsection we discuss the limitations for general spectroscopic systems. In order to estimate the importance of the first three problems listed above, we calculate in the Appendix the size of the first non-nkquantum selective operator from a $j$-order $n k$-quantum selective sequence, which is $\left(-i \bar{\zeta}^{(f+1)} t_{c}\right)_{\mathrm{nd}}$, and we determine plausible conditions for convergence of the average Hamiltonian expansion. Only the results will be summarized here. We combine phase cycling and symmetrization into one operation, which turns a $(j-2)$ order $n k$-quantum selective subcycle into a $j$-order $n k$ quantum selective cycle requiring $2 n$ subcycles [ $F i g$. 2(b)], assuming perfect phase shifts and no timing errors. The norm of the first nns term for the cycle, which is $\left(\overline{\mathcal{F C}}^{(+1)}\right)_{\text {nas }}$, is shown in the Appendix to be related to that of the first nns term of the $i$ th subcycle, which is $\left(\overline{\mathcal{H}}_{i}^{(j-1)}\right)_{\text {and }}$ :

$$
\begin{aligned}
& \left\|\left(\overline{\mathcal{H}}^{(j+1)} t_{c}\right)_{\text {mas }}\right\| \sim F(n)\left\|\left(\overline{\mathscr{F}}^{(0)} t_{c}\right)^{2}\right\|\left\|\left(\overline{\mathcal{F}}_{t}^{(j-1)} t_{c}\right)_{\text {aus }}\right\| \\
& F(n)=\left(\frac{8}{15} n^{5}-\frac{2}{3} n^{3}+\frac{2}{5} n\right)^{1 / 2} / 8 n^{3} \sim 0.09 n^{-1 / 2} .
\end{aligned}
$$

If a $(j-2)$-order selective subcycle were repeated $2 n$ times without phase shifting in between, the first nonselective term would be $\mathfrak{H}_{i, \text { ans }}^{(j-1)} t_{c}$; the first nonselective term in the $j$-order selective sequence is smaller than this only if $\left\|\left(\overline{\mathcal{F}}^{(0)} t_{c}\right)^{2}\right\| F(n)<1$. This result suggests that the average Hamiltonian expansion fails to converge when $\left\|\left(\overline{\mathscr{H}}^{(0)} t_{c}\right)^{2}\right\| \gtrsim F(n)^{-1}$. Thus, we require

$$
\left\|\left(\overline{\mathcal{K}}^{(0)} t_{c}\right)^{2}\right\|<F(n)^{-1} \text {. }
$$

Values of $F(n)$ are listed in Table III.

Equation (40) can be used $(j-1) / 2$ times, to express $\left(\overline{\mathcal{H}}^{(j+1)}\right)_{n \text { no }}$ in terms of $\left(\overline{\mathcal{F}}^{(2)}\right)_{\text {ans }}$ for a first-order selective sequence. $\left(\overline{\mathcal{H}}^{(2)}\right)_{\text {nns }}$ can then be calculated using Eq. (16). We define the selectivity $S$ of a $j$-order $n k$-quantum selective sequence to be the ratio between a typical ma-

TABLE III. Values of $F(n)=\left[(8 / 15) n^{5}\right.$ $\left.-(2 / 3) n^{3}+(2 / 15) n\right]^{1 / 2} / 8 n^{3}$ and $[F(n)]^{-1}$. As long as $\left\|\left(\overline{\mathscr{C}}^{(0)} t_{c}\right)^{2}\right\|<[F(n)]^{-1}$ the average Hamiltonian expansion is expected to converge rapidly.

\begin{tabular}{rlc}
\hline$n$ & $F(n)$ & $F(n)^{-1}$ \\
\hline 3 & 0.090 & 11 \\
4 & 0.044 & 23 \\
5 & 0.036 & 27 \\
6 & 0.032 & 31 \\
10 & 0.029 & 35 \\
12 & 0.026 & 38 \\
14 & 0.024 & 41 \\
16 & 0.023 & 44 \\
18 & 0.022 & 46 \\
\hline \hline
\end{tabular}




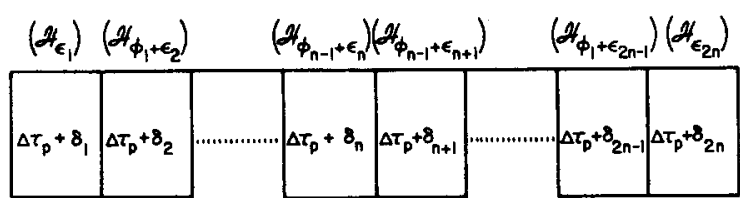

FIG. 9. Modification of the symmetrized sequence of Fig. 4(b) to include phase errors $\delta_{i}$ and timing errors $\epsilon_{i}$. We assume, without loss of generality, that $\left\langle\epsilon_{i}\right\rangle=\left\langle\delta_{i}\right\rangle=0$.

trix element of $\overline{\mathrm{HC}}^{(0)}$ and a typical matrix element of $\left(\overline{\mathcal{F}}^{(j+1)}\right)_{\text {ans }}$. At the limit of convergence $\left[\left\|\overline{\mathcal{H}}^{(0)} t_{c}\right\| \sim F(n)^{-1}\right]$ we find

$$
\begin{aligned}
S= & K^{-1} F(n)^{1 / 2}(2 n)^{\left(\mathrm{j}^{2} / 4+7 / 4\right)} \\
& \times\left\{\frac{n^{2} \alpha^{2}}{8 K}+\frac{n \alpha^{3}}{12}\left[F(n)\left\|\left(\overline{\mathcal{H}}^{(0)} t_{c}\right)^{3}\right\| /\left\|\overline{\mathcal{H}}^{(0)} t_{c}\right\|\right]^{2}\right\}^{-1 / 2},
\end{aligned}
$$

where $K$ is defined as the total number of allowed transitions divided by the total number of $n k$-quantum transitions, and $\alpha$ is defined by the relation $\left\|\mathcal{K}_{\phi}\right\|^{2}=\alpha K\left\|\overline{\mathcal{K}}^{(0)}\right\|^{2}$ [see $\mathrm{Eq} .(20)$; the reason for the definition is that if all the matrix elements of $\mathcal{H}_{\phi}$ have roughly equal magnitude, $\alpha \sim 1$.

To go further, we need to know the relative sizes of $\left\|\overline{\mathcal{K}}^{(0)}\right\|,\left\|\left(\overline{\mathcal{H}}^{(0)}\right)^{2}\right\|$, and $\left\|\left(\overline{\mathcal{H}}^{(0)}\right)^{3}\right\|$. If the eigenvalues of $\overline{\mathrm{K}}^{(0)}$ have a Gaussian distribution, we know from $\mathrm{Eq}$. (7) that $\left\|\left(\overline{F C}^{(0)}\right)^{2}\right\|=\sqrt{3}\left\|\overline{\mathcal{F}}^{(0)}\right\|^{2}$ and $\left\|\left(\overline{\mathcal{H C}}^{(0)}\right)^{3}\right\|=\sqrt{15}\left\|\overline{\mathcal{F}}^{(0)}\right\|^{3}$. Another possibility is that the energy levels might be spaced so that $\mathfrak{H}_{0}$ has only two transitions which are nearly resonant, forming an effective three-level system, and $\overline{\mathcal{H}}^{(0)}$ contains a nonzero matrix element for only one of these transitions; in this case $N_{T}=3$, and if $\overline{\mathcal{H}}^{(0)}$ is traceless, we expect $\left\|\left(\overline{\mathscr{K}}^{(0)}\right)^{n}\right\|=(3 / 2)^{(n-1) / 2}\left\|\overline{\mathscr{H}}^{(0)}\right\|^{n}$. In both cases $\left\|\left(\overline{\mathcal{K}}^{(0)}\right)^{n}\right\|$ does not grow greater than $\left.\| \overline{\mathcal{H}}^{(0)}\right) \|^{n}$ very rapidly. However, if $K \gg 1$, so that only a very small fraction of the matrix elements of $\mathfrak{H}_{\phi}$ are selected by $\overline{\mathcal{H}}^{(0)}$, we may have $\left\|\left(\overline{\mathcal{H}}^{(0)}\right)^{n}\right\| \sim\left(N_{T}\right)^{(n-1) / 2}\left\|\overline{\mathcal{H}}^{(0)}\right\|^{n}$; this case and the case of a Gaussian distribution will be discussed in the next section in connection with multiplequantum NMR.

The factor $(2 n)^{y^{2 / 4+7 / 4}}$ makes $S$ grow very rapidly as $j$ is increased, and fairly small values of $j$ still give very selective sequences. For example, if a Gaussian distribution of eigenvalues is assumed for $\overline{\mathcal{H}}^{(0)}$, the selectivity of a third-order $10 k$-quantum selective sequence with $\alpha \sim 1, K \sim n$, and $F(n)=0.028$ (from Table III) is $S=1170$; a typical selected matrix element is more than three orders of magnitude larger than a typical nonselected matrix element, even near, the limit of convergence. When $\left\|\left(\overline{\mathcal{H C}}^{(0)} t_{c}\right)^{2}\right\| \ll F(n)^{-1}, S$ will be much larger; in general, if $\left\|\left(\overline{\mathcal{K}}^{(0)} t_{c}\right)\right\|$ is scaled down by a factor of $A,\left\|\overline{\mathcal{H}}^{(n+1)} t_{c}\right\|_{\text {me }}$ is scaled down by a factor of $A^{j+2}$, and $S$ increases by a factor of $A^{j+1}$. We conclude that for many systems the use of cycles with only a finite order of selectivity is entirely satisfactory.

The effects of timing errors and imperfect phase shifts are more serious. Suppose that the length of subcycle $i$ is $\Delta \tau_{p}+\delta_{i}$, and that the phase is $\phi_{i}+\epsilon_{1}$, where $\sum_{1} \delta_{i}$ $=\sum_{i} \epsilon_{i}=0$ (Fig.' 9 ). Then

$$
\begin{aligned}
\overline{\mathcal{H}}^{(0)}= & \frac{1}{2 n \Delta T_{p}} \sum_{i}\left(\Delta \tau_{p}+\delta_{i}\right) \\
& \times \exp \left[i I_{i}\left(\phi_{i}+\epsilon_{i}\right)\right] \mathcal{H}_{0} \exp \left[-i I_{z}\left(\phi_{i}+\epsilon_{i}\right)\right],
\end{aligned}
$$

which is no longer purely $n k$-quantum selective; the matrix element for an $m$-quantum transition is multiplied by

$$
\frac{1}{2 n \Delta \tau_{p}} \sum_{j}\left(\Delta \tau_{p}+\delta_{j}\right) \exp \left[i m\left(\phi_{j}+\epsilon_{j}\right)\right]
$$

instead of 0 . Assuming $\delta_{j} \ll \Delta \tau_{p}$ and $\epsilon_{j} \ll 1$, we can expand this out:

$$
\begin{aligned}
\frac{1}{2 n \Delta \tau_{p}} & \sum_{i}\left(\Delta \tau_{p}+\delta_{i}\right) \exp \left[i m\left(\phi_{i}+\epsilon_{i}\right)\right] \\
= & \frac{1}{2 n \Delta \tau_{p}}\left[\sum \Delta \tau_{p} \exp \left(i m \phi_{i}\right)+\sum \delta_{i} \exp \left(i m \phi_{i}\right)\right. \\
& \left.+\sum\left(\Delta \tau_{p}\right)\left(i m \epsilon_{i}\right) \exp \left(i m \phi_{i}\right)+\cdots\right] .
\end{aligned}
$$

The first term on the rhs corresponds to an ideal sequence and vanishes if $m$ is not a multiple of $n$. If $\epsilon_{i}$ and $\delta_{i}$ are uncorrelated with $\phi_{i}$, the last two terms reduce to $(2 n)^{-1 / 2}\left[\left(\left\langle\delta_{j}^{2}\right\rangle / \Delta \tau_{p}^{2}\right)+m^{2}\left\langle\epsilon_{j}^{2}\right\rangle\right]^{1 / 2}$. If the number of subcycles increases (for example, by going to a higher-order selective sequence), this term decreases, so that the ratio $\left\|\overline{\mathcal{K}}^{(0)}\right\|_{s e l e c t i v e} /\left\|\overline{\mathcal{K}}^{(0)}\right\|_{\text {mas }}$ can be made ar bitrarily large. However, if $\epsilon_{j}$ or $\delta_{j}$ are completely correlated with $\phi_{i}$ (so that, for example, every time the phase should be $\phi=0$ it is actually $\left.\phi=\epsilon_{0}\right),\left\|\overline{\mathcal{F}}^{(0)}\right\|_{\text {elective }} /$ $\left\|\overline{\mathcal{F C}}^{(0)}\right\|_{\mathrm{nm}}$ is not reduced by increasing the order of the sequence. Such a situation arises with a miscalibrated phase shifting device or for one in which a digital approximation is made to the exact setting. One way to reduce this error is to use two (or more) phase shifting devices, so that the total error is not well correlated with the total phase.

\section{B. Application of nonideal selective sequences to multiple-quantum NMR}

As mentioned earlier, an ideal $N$-quantum sequence that had no zero-quantum matrix elements could enhance the single $N$-quantum transition of an $N$-spin system by a factor of $N 2^{N}$. A nonideal $n k$-quantum selective sequence (which has zero-quantum matrix elements) will not work as well, for two reasons. First, there may be nonzero matrix elements for the populations of the states $m= \pm N / 2$ (populations may be thought of as a special type of zero-quantum coherence, with the initial state identical to the final state). In this case, the effective Hamiltonian for the two levels will be $a_{x} I_{x}^{a b}+$ $a_{y} I_{y}^{a b}+a_{z} I_{z}^{a b}$ (Fig. 10) instead of containing only $I_{x}^{a b}$ and $I_{y}^{a b}$. Depending on the relative size of the coefficient $a_{k}$, it may be impossible to transfer population completely into coherence. Statistically, we expect the coefficients to be of comparable size and in that case much of the population can be transferred into coherence.

A much more serious effect comes from the requirement that the average Hamiltonian expansion converge. In general, this would imply $\left\|\left(\overline{\mathcal{F}}^{(0)} t_{c}\right)^{2}\right\| \ll F(n)^{-1}$. For the sequences described, the distribution of eigenvalues 


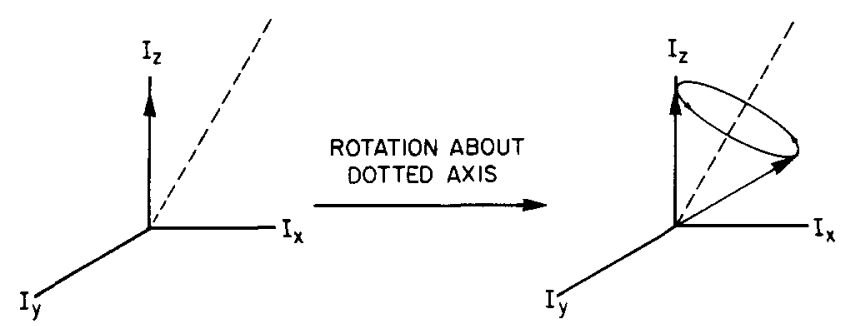

FIG. 10. Schematic illustration of one effect of zero-quantum coherences on an $N$-quantum selective sequence. The sequence causes the population difference between the states $m= \pm N / 2$ to be rotated about an arbitrary axis, and complete transfer of population difference into coherence may be impossible. Compare this to Fig. 8 .

of $\mathcal{K}_{\phi}$ is expected to resemble that of the eigenvalues of $\mathcal{F}_{D, k a}$, which will be Gaussian if $N$ is large. It is reasonable to assume that the eigenvalues of $\overline{\mathfrak{H}}^{(0)}$ have a Gaussian distribution, since $\overline{\mathscr{H}}^{(0)}$ has many allowed zero-quantum transitions, and since the limit of $\overline{\mathcal{H}}^{(0)}$ as

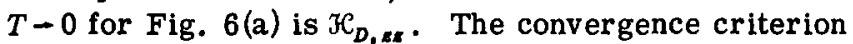
of Eq. (41) can then be written as $\left\|\overline{\mathcal{H}}^{(0)} t_{c}\right\| \ll 3^{-1 / 4} F(n)^{-1 / 2}$ $\sim 2.1\left(n^{1 / 4}\right)$.

From the definition of the norm of a $2^{N}$ by $2^{N}$ matrix we find

$$
\begin{aligned}
\left\|\overline{\mathcal{H}}^{(0)} t_{c}\right\| & =\left\{2^{-N} \operatorname{tr}\left[\left(\overline{\mathcal{K}}^{(0)} t_{c}\right)^{2}\right]\right\}^{1 / 2} \\
& =\left(2^{-N} \sum_{m n}\left|\overline{\mathcal{K}}^{(0)} t_{c}\right|_{m n}^{2}\right)^{1 / 2} \\
& =\sqrt{N_{s}} 2^{-N / 2}\left\langle\left|\overline{\mathscr{K}}^{(0)} t_{c}\right|_{m n}^{2}\right\rangle^{1 / 2},
\end{aligned}
$$

where $N_{s}$ is the number of possibly nonzero matrix elements, and the rms average only includes those elements. Therefore, to make an average excited matrix element comparable to 1 (which is needed if we want the effect of $\overline{\mathrm{H}}^{(0)}$ to approximate a selective $90^{\circ}$ pulse between the levels $m= \pm N / 2$ ) we need

$$
N_{s} \leq 2^{N} \text {. }
$$

There are $2^{2 N}$ possibly nonvanishing matrix elements for a nonselective sequence, and if only zero-quantum and $N$-quantum elements are excited, $N_{S} \sim 2^{2 N} N^{-1 / 2}$. For $N \geq 2$, Eq. (46) cannot be satisfied. Assuming $\left\|\overline{\mathcal{H}}^{(0)} t_{c}\right\| \sim 1$, we expect

$$
\left\langle\left(\overline{\mathcal{H C}}^{(0)} t_{c}\right)_{a b}^{2}\right\rangle^{1 / 2}=2^{N / 2} / N^{-1 / 4} 2^{N} \ll 1 .
$$

This scales down the possible gain because the selective sequence effectively produces only a small rotation instead of $a 0^{\circ}$ pulse. In fact,

$$
\begin{aligned}
& \left|\left(U I_{z} U^{\dagger}\right)_{a b}\left(V I_{g} V^{\dagger}\right)_{b a}\right| \sim\left(N^{1 / 4 / 2^{N / 2}}\right)^{2}\left(I_{s}\right)_{m=N / 2}^{2} \\
& =\left(\sqrt{N} 2^{-N}\right)\left(\frac{N^{2}}{4}\right) \text {, }
\end{aligned}
$$

so the gain is

$$
G^{\prime}=\left[\beta \sqrt{N} 2^{-N}\left(I_{s}^{2}\right)_{m=N / 2}\right] / \beta 2^{-2 N} \operatorname{tr}\left(I_{\varepsilon}^{2}\right)=N^{3 / 2} .
$$

This value of $G^{\prime}$ is only approximate, since it depends on the exact maximum permissible value of $\left\|\overline{\mathcal{K}}^{(0)} t_{c}\right\|$. The gain can be assinged to two effects. Only $\sim 1 / \sqrt{N}$ as many transitions are being pumped so each one is $\sqrt{N}$ times stronger; in addition, the $N$-quantum transition receives intensity from the equilibrium population difference of the extreme states for which the expectation value of $I_{2}^{2}$ is $N^{2} / 4$, a factor of $N$ greater than the expectation value averaged over all states. While this gain is large, a much larger gain is possible if $N_{S}$ can be reduced.

\section{Removal of O-quantum operators from selective sequences}

The gain can be increased if the zero-quantum coherences are removed from $\overline{\mathcal{H}}^{(0)}$. One way to do this is with the sequence shown in Fig. 11(a). The phase shift of $\pi / N$ inverts the $N$-quantum coherence but leaves the zero-quantum coherence invariant, and the time re-

(a)

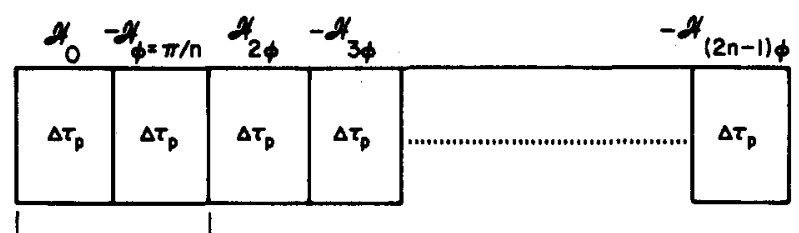

(b)
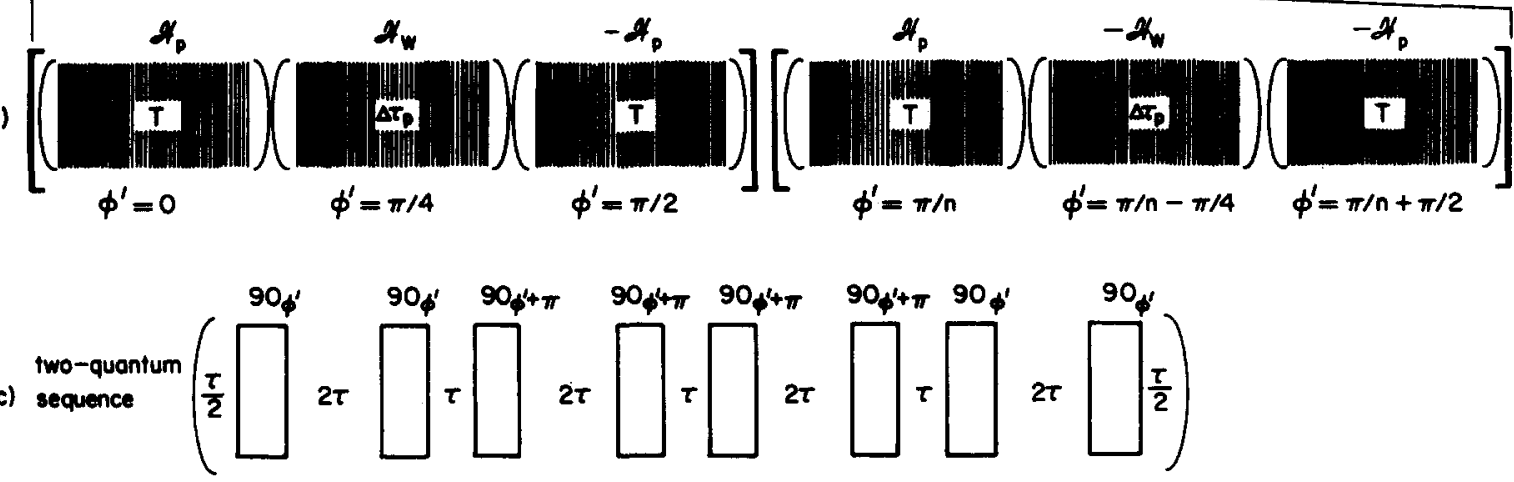

FIG. 11. Sequences to select only $N$-quantum, $3 N$-quantum, $\ldots,(2 k+1) N$-quantum coherences. (a) General sequence. Note that $\mathfrak{K}_{\infty}$ is inverted after every subcycle, and that the phase shift is $\phi=\pi / N$, instead of $\phi=2 \pi / N$ as in Fig. 4 . (b) $\mathfrak{K}_{p}$ and $\mathfrak{K}_{w}$ can be formed with the pure double-quantum sequence [part (c)], which is inverted by a $\pi / 2$ phase shift. 
versal inverts every order of coherence, so the net result is that zero-quantum coherences are inverted every subcycle but $N$-quantum coherences are unaffected. The lowest-order average $\overline{\mathcal{H}}^{(0)}$ for the sequence in Fig. 11(a) contains only $N$-quantum, $3 N$-quantum, $\ldots,(2 k+1) N-$ quantum coherences after $2 N$ subcycles. In an $N$-spin system, this makes $N_{s} \ll 2^{N}$, condition (46) is satisfied, and the ideal gain $G_{N}=N 2^{N}$ of Table I becomes possible.

The easiest way to design such a sequence for $\mathfrak{H}_{\phi}$ is shown in Fig. 11(b). $\mathfrak{K}_{p},-\mathfrak{K}_{p}, \mathfrak{K}_{w}$, and $-\mathfrak{K}_{w}$ are all generated from the double-quantum sequence, mentioned earlier and illustrated in Fig. 11 (c) with relative phases $0, \pi / 2, \pi / 4$, and $3 \pi / 4$, respectively. If $\left\|\mathcal{K}_{w} \Delta \tau_{p}^{\prime}\right\| \ll 1$, only the $N$-quantum transition appears. However, the value of $K=\left\|\mathfrak{H}_{\phi}\right\| /\left\|\overline{\mathcal{H}}^{(0)}\right\|$ is now very large $\left(K \sim 2^{N}\right)$ so the selectivity $S$ from Eq. (41) will be somewhat weaker, and should be reconsidered. High-order selective sequences with no zero-quantum contributions can be generated from Fig. 11(a) in exactly the same way that high-order selective sequences with zero-quantum contributions were generated from Fig. 4(a). A third-order 10 -quantum selective sequence requires $(4 N)^{2}=1600 \mathrm{sub}-$ cycles [instead of $(2 N)^{2}=400$ subcycles without suppres sion of zero quantum]. Equation (41) applies if $(2 N)^{\gamma^{2} / 4+7 / 4}$ is replaced with $(4 N)^{f^{2} / 1+7 / 4}$ and $N$ is replaced with $2 N$, since each symmetrized phase cycling now requires $4 N$ subcycles.

The assumption of a Gaussian distribution of eigenvalues is no longer valid, since $\overline{\mathcal{H}}^{(0)}$ has only two nonzero matrix elements. Since $\overline{\bar{x}}^{(0)}$ is Hermitian, the two elements have the same magnitude $\frac{1}{2} R$. The eigenvalues are $\pm \frac{1}{2} R$, and 0 for all other states, because $\overline{\mathcal{H}}^{(0)}$ is traceless. Therefore,

$$
\begin{aligned}
\left\|\overline{\mathcal{K}}^{(0)} t_{c}\right\|= & {\left[\left(2^{-N}\right)(2)\left(R^{2} / 4\right)\right]^{1 / 2}=R 2^{-(N+1) / 2}, } \\
\left\|\left(\overline{\mathcal{K}}^{(0)} t_{c}\right)^{2}\right\| & =\left[\left(2^{-N}\right)(2)\left(R^{4} / 16\right)\right]^{1 / 2} \\
& =R^{2} 2^{-(N+3) / 2}=2^{(N-1) / 2}\left\|\overline{\mathcal{H}}^{(0)} t_{c}\right\|^{2}, \\
\left\|\left(\overline{\mathcal{K}}^{(0)} t_{c}\right)^{3}\right\| & =\left[\left(2^{-N}\right)(2)\left(R^{6} / 64\right)\right]^{1 / 2} \\
& =R^{3} 2^{-(N+5) / 2}=2^{(N-1)}\left\|\overline{\mathcal{H}}^{(0)} t_{c}\right\|^{3} .
\end{aligned}
$$

In the limit of convergence $\left[\left\|\left(\overline{\mathcal{F C}}^{(0)} t_{c}\right)^{2}\right\| \sim F(n)^{-1}\right]$ we find the calculated selectivity of a third-order 10-quantum sequence with $\alpha=1, K=2^{2 N-1}$, and $F(n)=0.029$ [see Eq. (41)] to be $S=0.025$. However, we do not really need to have $\left\|\left(\overline{\mathcal{K}}^{(0)} t_{c}\right)^{2}\right\|$ be this large; all we really want is $R t_{c}=\pi / 2$, so $\left\|\left(\overline{\mathcal{H}}^{(0)} t_{c}\right)^{2}\right\|=0.027$, a factor of 1797 smaller, When $\left\|\left(\bar{K}^{(0)} t_{c}\right)^{2}\right\|$ has this value, we find $S=8.0 \times 10^{4}$.

We can attain the maximum gain $G$ if the $4\left(2^{N}-2\right)$ non- $N$-quantum selective matrix elements that involve the $m= \pm N / 2$ states transfer only a small fraction of $\left(I_{n}^{2}\right)_{m \rightarrow N / 2}$ relative to the fraction transferred by the two $N$-quantum selective matrix elements. Since each selective matrix element is larger by a factor $S$, the intensity of the selected transition [from Eq. (32)] is larger by a factor $S^{2}$, and we require

$$
\begin{aligned}
& 2 S^{2} \gg 4\left(2^{N}-2\right), \\
& S^{2} \gg 2^{N+1}-4,
\end{aligned}
$$

which is satisfied in this example. We therefore con- clude almost all the theoretical gain from an infiniteorder selective sequence is attainable with a third-order selective sequence, and the potential gains in Table I should be approximately realizable.

Note that the theoretical maximum gain becomes more difficult to achieve as $N$ increases, for several reasons. From Eq. (53) we see that the required selectivity for maximum gain is proportional to $(\sqrt{2})^{N}$, and therefore high-order selective sequences may be needed. How ever, the number of subcycles cannot be increased indefinitely, because each subcycle must have a minimum duration, and relaxation effects limit the maximum duration of the cycle to less than $T_{2}$. Typically, $\left\|\mathcal{K}_{D_{1}, E}\right\|$ $\sim 10 \mathrm{kHz}$ and $T_{2} \sim 100 \mathrm{~ms}$ for liquid crystal systems, so no more than a few thousand subcycles would be possible; a third-order 18-quantum selective sequence that eliminates zero-quantum requires $(4 n)^{2}=5184$ subcycles, and therefore is impossible for many molecules. In addition, sample heating becomes a serious problem when many pulses are applied.

\section{CONCLUSION}

On the basis of the calculations presented in this paper and experimental results reported elsewhere, 7,17 we believe that selective excitation will increase the number and size of molecules which can be studied by multiple-quantum NMR. We also expect that this approach to selective excitation of coherence and selective population inversion will be useful in a variety of systems.

\section{ACKNOWLEDGMENTS}

We wish to thank Gary Drobny, Dr. Luciano Mueller, James Murdoch, Steve Sinton, and Jau Tang for stimulating discussions, and Terry Judson for her patience in typing the manuscript. W.S.W. holds a National Science Foundation Graduate Fellowship. This research was supported by the Division of Materials Sciences, Office of Basic Energy Sciences, U.S. Department of Energy under contract No. W-7405-Eng-48.

\section{APPENDIX}

In this Appendix we calculate the size of the first non$n k$-quantum selective operator from a $j$-order $n k$-quantum selective sequence, which is $\left(-\overline{w e}^{(j+1)} t_{c}\right)_{\text {nns }}$.

To simplify the calculations we combine phase cycling and symmetrization into one operation, which turns a $(j-2)$-order $n k$-quantum selective subcycle ( $j$ odd) into a $j$-order $n k$-quantum selective cycle requiring $2 n$ subcycles [Fig. 4(b)], assuming perfect phase shifts and no timing errors. This will allow us to write $\overline{\mathcal{K}}^{(j+1)}$. As in our earlier calculations, we expand the propagator for the $j$-order $n k$-quantum selective sequence in powers of $t_{c}$. The first nonselective term is $\overrightarrow{i \bar{F}^{(j+1)}} t_{c}$, which is proportional to $t_{c}^{(j+2)}$. All other terms proportional to $t_{c}^{j+2}$ are $n k$-quantum selective, so the only possible nns term proportional to $t_{c}^{(j+2)}$ is $\left(i \overline{\mathcal{T C}}^{(j+1)} t_{c}\right)_{\mathrm{ase}}$. The product of the propagators for the subcycles has several terms proportional to $\left(t_{c i}\right)^{j+2}=\left(t_{c} / 2 n\right)^{j+2}$; they are 


$$
\begin{aligned}
& \prod_{i} U\left(t_{c i}\right)^{i}=-i \sum_{i} \overline{\mathcal{F}}^{(j+1)} t_{c i} \\
& -\sum_{i<j}\left(\overline{\mathcal{K}}_{i}^{(0)} \overline{\mathcal{K}}_{j}^{(j)}+\overline{\mathcal{K}}_{i}^{(j)} \overline{\mathcal{K}}_{j}^{(0)}\right) t_{c i}^{2} \\
& -\frac{1}{2} \sum_{i}\left(\overline{\mathscr{K}}_{i}^{(0)} \overline{\mathcal{K}}_{i}^{(j)}+\overline{\mathscr{K}}_{i}^{(j)} \overline{\mathcal{K}}_{i}^{(0)}\right) t_{c i}^{2} \\
& -\sum_{i<j}\left(\overline{\mathcal{K}}_{i}^{(1)} \overline{\mathcal{K}}_{j}^{(j-1)}+\overline{\mathcal{K}}_{i}^{(j-1)} \overline{\mathcal{H}}_{j}^{(1)}\right) t_{c i}^{2} \\
& -\frac{1}{2} \sum_{i}\left(\overline{\mathcal{F}}_{i}^{(1)} \overline{\mathcal{K}}_{i}^{(j-1)}+\overline{\mathcal{H}}_{i}^{(j-1)} \overline{\mathcal{K}}_{i}^{(1)}\right) t_{c i}^{2} \\
& +\left[i \sum_{i>j>k} t_{c i}^{3}+(i / 2) \sum_{i>j>k} t_{c i}^{3}+(i / 2) \sum_{i>j=k} t_{c i}^{3}\right]
\end{aligned}
$$

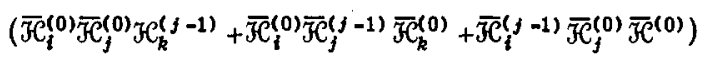

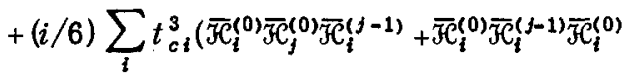

$$
\begin{aligned}
& \left.+\overline{\mathcal{K}}_{i}^{(j-1) \overline{\mathcal{H}}_{i}^{(0)}} \overline{\mathcal{H}}_{i}^{(0)}\right) \text {. }
\end{aligned}
$$

+ terms which are not proportional to $\left(t_{c}\right)^{j+2}$. We will assume that the subcycles are themselves constructed by phase cycling and symmetrization, so that $\overline{\mathfrak{H}}_{i}^{\left(j \pm 2 j^{\prime \prime}\right)}=0$ for all $j^{\prime}$, and terms (A2)-(A5) vanish. In addition, terms (A1) and (A7) are unaffected by a phase shift of $\phi=2 \pi / n$, so they are $n k$-quantum selective. Finally, term (A6) contains many $n k$-quantum selective portions; for example, $\sum_{i=j<k} \overline{\mathfrak{H}}_{j}^{(0)} \overline{\mathfrak{F}}_{j}^{(0)} \overline{\mathcal{H}}_{k}^{(j-1)}$ is $n k$-quantum selective if $i$ and $j$ are in the first half of the cycle and $k$ is in the second half. As another example, if $i=j=1$, the nns part of the summation $\sum_{k>1} \overline{\mathcal{H}}_{1}^{(0)} \overline{\mathcal{H}}_{1}^{(0)} \overline{\mathcal{K}}_{k}^{(j-1)}$, which contains $(n-1)$ terms, is the same size as a single term, since $\left[\left(\overrightarrow{\mathcal{F}}_{1}^{(0)} \overline{\mathcal{K}}_{1}^{(0)}\right)\left(\sum_{k} \overline{\mathcal{H}}_{k}^{(j-1)}\right)\right]_{\text {nns }}=0$. Straightforward but tedious counting of the remaining terms, assuming that their matrix elements add randomly, gives

$$
\begin{aligned}
& \left.\left\|\left(\overline{\mathcal{F}}^{(j+1)} t_{c}\right)\right\|_{\text {nns }}^{2} \sim F(n)^{2\langle}\left\|\left(\overline{\mathcal{K}}_{i}^{(0)}\right)^{2} \overline{\mathcal{F}}_{j}^{(j-1)}\right\|\right\rangle^{2} t_{c}^{6}, \\
& F(n)=\left(\frac{8}{15} n^{5}-\frac{2}{3} n^{3}+\frac{2}{15} n\right)^{1 / 2} / 8 n^{3} \sim 0.09 n^{-1 / 2} .
\end{aligned}
$$

The construction of the subcycles implies $\overline{\mathfrak{H}}_{i}^{(0)}=\overline{\mathcal{H}}_{j}^{(0)}$ and $\left\|\overline{\mathcal{K}}_{i}^{(j-1)}\right\|=\left\|\overline{\mathcal{H}}_{j}^{(j-1)}\right\|$, and therefore $\left\langle\left\|\left(\overline{\mathcal{K}}_{i}^{(0) 2}\right) \overline{\mathcal{F}}_{j}^{(j-1)}\right\|^{2}\right\rangle$ $\sim\left\langle\left\|\overline{\mathcal{K}}_{i}^{(0)} \overline{\mathcal{K}}_{j}^{(j-1)} \overline{\mathcal{F}}_{k}^{(0)}\right\|^{2}\right\rangle \sim\left\langle\left\|\overline{\mathcal{H}}_{i}^{(j-1)}\left(\overline{\mathcal{F}}_{j}^{(0)}\right)^{2}\right\|^{2}\right\rangle$. We expect to find

$$
\left\|\left(\overline{\mathcal{H}}^{(0)}\right)^{2} \overline{\mathcal{F}}_{\text {nns }}^{(j-1)}\right\| \sim\left\|\left(\overline{\mathcal{F}}^{(0)}\right)^{2}\right\|\left\|\overline{\mathcal{F}}_{j, n n s}^{(j-1)}\right\|
$$

so

$$
\left\|\overline{\mathcal{F}}_{\mathrm{nns}}^{(j+1)} t_{c}\right\| \sim F(n)\left\|\left(\overline{\mathcal{F}}^{(0)} t_{c}\right)^{2}\right\|\left\|\overline{\mathcal{Y}}_{j_{, \text {nas }}}^{(j-1)} t_{c}\right\| .
$$

Since $\overline{\mathcal{H}}^{(J)}$ is proportional to $t_{c}^{j}$, we expect that if $t_{c}$ is "small," $\left\|\overline{\mathcal{F}}^{(j+1)}\right\| \gg\left\|\overline{\mathcal{H}}^{(j+3)}\right\|$ (we assume the cycles are symmetrized, so that $\overline{\mathfrak{K}}^{(j+2)}$ vanishes) and if $t_{c}$ is "large," $\left\|\overline{\mathcal{F}}^{(j+1)}\right\| \ll\left\|\overline{\mathcal{F}}^{(j+3)}\right\|$. We are interested in finding the value of $t_{c}$ which makes $\left\|\overline{\mathcal{H}}^{(j+1)}\right\| \sim\left\|\overline{\mathcal{H}}^{(j+3)}\right\|$; if we make $t_{c}$ much smaller than this critical value, we expect to find convergence.

Assume that we have a $(j-2)$-order $n k$-quantum selective subcycle which is known to converge, so that $\left\|\overline{\mathcal{F}}^{(0)}\right\| \gg\left\|\overline{\mathcal{K}}_{j}^{(2)}\right\|$ and $\left\|\overline{\mathcal{K}}_{i}^{(j-1)}\right\|_{\text {ms }} \gg\left\|\overline{\mathcal{F}}_{i}^{(j+1)}\right\|_{\text {nns }}$. To create a $j$-order $n k$-quantum selective cycle we need to increase the cycle time by a factor of $2 n$. We have calculated $\left\|\mathcal{H}^{(j+1)}\right\|_{n \mathrm{~nm}}$ by examining the term in the propagator proportional to $t_{c}^{j+2}$. The largest nns terms pro- portional to $t_{c}^{j+4}$, under these assumptions, are

$$
\begin{aligned}
& \left\{-i \overline{\mathcal{K}}^{(j+3)} t_{c}+\frac{1}{2} i t_{c}^{3}\left[\left(\overline{\mathcal{K}}^{(j+1)}\right)\left(\overline{\mathcal{K}}^{(0)}\right)^{2}+\overline{\mathcal{H}}^{(0)} \overline{\mathcal{K}}^{(j+1)} \overline{\mathcal{K}}^{(0)}\right.\right.
\end{aligned}
$$

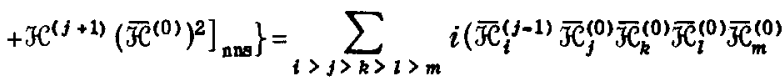

+ permutations) $)_{\mathrm{nms}} t_{c i}^{5}+$ small terms.

Again a substantial fraction of the rhs cancels or is forced to be selective. Most of the remaining terms on the rhs cancel with terms such as $\overline{\mathcal{H}}^{(j+1)}\left(\overline{\mathcal{H}}^{(0)}\right)^{2}$ on the lhs. The result is that, if $\left\|\left(\overline{\mathcal{F}}^{(0)} t_{c}\right)^{2}\right\| \sim 1$, we expect to find $\left\|\overline{\mathcal{H}}^{(j+3)}\right\|_{\mathrm{nns}}<\left\|\mathcal{H}^{(j+1)}\right\|_{\mathrm{nns}}$. Thus, if we have a converging $(j-2)$-order $n k$-quantum selective sequence, we expect to retain convergence for the $j$-order selective sequence if $\left\|\overline{\mathcal{K}}^{(0)} t_{c}\right\| \ll F(n)^{-1}$.

As mentioned, we can use Eq. (A9) iteratively, to calculate the first nns term as a function of successively lower-order terms. To begin the iteration, however, we need an expression for $\left\|\overline{\mathcal{K}}^{(2)}\right\|_{n \text { m }}$ for a first-order selective sequence [Fig. 4(b) with $\mathfrak{H}_{\phi}$ nonselective]. We have shown that $[\mathrm{Eq} .(16)]$

$$
\begin{aligned}
& \left(\overline{\mathcal{F}}^{(2)}\right)_{\text {uns }}=\left(i / t_{c}\right)\left[\int_{t_{3}=0}^{t_{c}} d t_{3} \int_{t_{2}=0}^{t_{3}} d t_{2}\right. \\
& \left.\times \int_{t_{1}=0}^{t_{2}} d t_{1} \tilde{\mathcal{H}}_{1 \mathrm{in}}\left(t_{3}\right) \tilde{\mathcal{H}}_{\mathrm{int}}\left(t_{2}\right) \tilde{\mathcal{H}}_{1 \mathrm{tnt}}\left(t_{1}\right)\right]_{\mathrm{nns}} .
\end{aligned}
$$

$\tilde{\mathfrak{H}}_{\text {int }}$ in this expression is $\mathfrak{H}_{\phi}$, as discussed in relation to Fig. 5. We can rewrite this as

$$
\begin{aligned}
\left(\overline{\mathcal{H}}^{(2)}\right)_{\mathrm{nas}}= & \left(-i / 2 n \Delta \tau_{p}\right)\left(\Delta \tau_{p}^{3}\right) \\
& \times\left(\sum_{i<j<k}+\frac{1}{2} \sum_{i=j<k}+\frac{1}{6} \sum_{i=f=k}\right) \\
& \times\left(\mathfrak{H C}_{\phi(i)} \mathfrak{H C}_{\phi(j)} \mathfrak{H C}_{\phi(k)}\right)_{\text {nns }},
\end{aligned}
$$

where $\phi(i)$ is the value of $\phi$ during the $i$ th subcycle. Since the operators $\mathcal{H}_{\phi(i)}$ are nonselective, only a few terms in the summations are forced to be $n k$-quantum selective (note, however, the sum with $i=j=k$ is $n k$ quantum selective, as are a few of the terms in the other sums). We divide the possible values of $i, j$, and $k$ into two sets:

(a) $i$ and $j$ are both in the first half of the cycle and $k$ is in the second half, or $i$ is in the first half and $j$ and $k$ are both in the second half. In this case the sum over the isolated index reduces to $n \overline{\mathrm{K}}^{(0)}$.

(b) $i, j$, and $k$ are all in the same half of the cycle. In this case no further reduction is possible. By construction of the sequence, $\left\|\mathcal{H}_{\phi_{(1)}}\right\|=\left\|\mathcal{K}_{\phi(1)}\right\|$. If we as sume $\left\langle\left\|\overline{\mathcal{F}}^{(0)} \mathcal{H}_{\phi_{(i)}} \mathcal{H}_{\Phi_{(j)}}\right\|\right\rangle=\left\langle\left\|\mathcal{H}_{\Phi(f)} \mathcal{K}_{\Phi} \overline{\mathcal{K}}^{(0)}\right\|\right\rangle$, straightforward algebra then gives

$$
\begin{aligned}
& \left\|\overline{\mathcal{H}}^{(2)} \Delta \tau_{p}\right\|_{n \mathrm{~ns}}^{2} \sim \frac{n}{12}\left\langle\left\|\mathcal{K}_{\phi_{(j)}} \mathcal{H}_{\Phi_{(j)}} \mathcal{K}_{\Phi_{(k)}} \Delta \tau_{p}^{3}\right\|^{2}\right\rangle \\
& \quad+\frac{n^{2}}{8}\left\langle\left\|\overline{\mathcal{H}}^{(0)} \mathcal{K}_{\phi_{(j)}} \mathcal{H}_{\Phi_{(k)}}\right\|^{2}\right\rangle .
\end{aligned}
$$

We expect $\left\|\overline{\mathcal{K}}^{(0)} \mathcal{H C}_{\phi(i)} \mathcal{H C}_{\phi(j)}\right\| \sim\left\|\overline{\mathcal{C}^{(0)}}\right\|\left\|\mathcal{H}_{\phi(i)} \mathcal{K C}_{\phi(j)}\right\|$ $\leq\left\|\overline{\mathcal{H}}^{(0)}\right\|\left\|\left(\mathcal{H C}_{\phi(i)}\right)^{2}\right\|$ and $\left\|\mathcal{H}_{\phi(i)} \mathcal{H}_{\phi_{(j)}} \mathfrak{H C}_{\phi_{(k)}}\right\| \leq\left\|\left(\mathcal{K C}_{\phi(i)}\right)^{3}\right\|$. 
Let $\left\|\mathcal{H}_{\phi(1)}\right\|^{2}=\alpha K\left\|\overline{\mathcal{H}}^{(0)}\right\|^{2}$ (see Sec. IV.A). We can now write

$$
\begin{aligned}
\left\|\overline{\mathcal{K}}^{(2)} \Delta \tau_{p}\right\|_{n \mathrm{~ns}} & \lesssim\left[\frac{n \alpha^{3} K^{3}}{12}\left\|\left(\overline{\mathcal{K}}^{(0)} \Delta \tau_{p}\right)^{3}\right\|^{2}\right. \\
& \left.+\frac{n^{2} \alpha^{2} K^{2}}{8}\left\|\left(\overline{\mathcal{K}}^{(0)} \Delta \tau_{p}\right)^{2}\right\|^{2}\left\|\overline{\mathcal{F}}^{(0)} \Delta \tau_{p}\right\|^{2}\right]^{1 / 2} .
\end{aligned}
$$

We want the first-order sequence to converge. If $\left\|\left[\mathcal{H}_{*}\left(2 n \Delta \tau_{p}\right)\right]^{2}\right\| \sim 1$, we see that $\left\|\overline{\mathcal{H}}^{(2)} \Delta \tau_{p}\right\|_{\text {nas }} \ll 1$. Combining this with our earlier results, we expect a $j$-order $n k$-quantum selective sequence which is constructed from $\frac{1}{2}(j+1)$ phase cycles and $\frac{1}{2}(j+1)$ symmetrizations [and therefore has a cycle time $\left.t_{c}=(2 n)^{(j+1) / 2} \Delta \tau_{p}\right]$ to converge if

$$
\|\left[\left(\mathfrak{H C}_{\phi}\left(2 n \Delta \tau_{p}\right)\right]^{2} \| \lesssim 1 \text { and }\left\|\left(\overline{\mathcal{F}}^{(0)}(2 n)^{(j+1) / 2} \Delta \tau_{p}\right)^{2}\right\| \ll F(n)^{-1}\right. \text {. }
$$

The size of the first nonselective term $\left\|\mathcal{K}^{(j+1)}\right\|_{n \text { ss }}$ is

$$
\begin{aligned}
& \left\|\overline{\mathcal{K}}^{(j+1)}\right\|_{\mathrm{nas}}(2 n)^{(j+1) / 2} \Delta \tau_{p} \lesssim F(n)\left\|\overline{\mathcal{F}}_{i}^{(j-1)}\right\|_{\mathrm{nns}}\left\|\left(\overline{\mathcal{K}}^{(0)}\right)^{2}\right\|\left(\Delta \tau_{p}\right)^{3}(2 n)^{3(j+1) / 2}\left\|\overline{\mathcal{H}}^{(j+1)}\right\|_{n \text { ns }} \lesssim F(n)\left\|\overline{\mathcal{H}}_{i}^{(j-1)}\right\|_{\mathrm{nns}}\left\|\left(\overline{\mathcal{K}}^{(0)} \Delta \tau_{p}\right)^{2}\right\|(2 n)^{j+1} \\
& =F(n)^{2}\left\|\overline{\mathcal{H}}_{i}^{(j-3)}\right\|_{\text {ans }}\left\|\left(\overline{\mathcal{H}}^{(0)} \Delta \tau_{p}\right)^{2}\right\|^{2}(2 n)^{(j+1)+(j-1)}=[F(n)]^{(j-1) / 2}\left\|\overline{\mathcal{K}}_{i}^{(2)}\right\|_{\text {nas }}\left\|\left(\overline{\mathcal{K}}^{(0)} \Delta \tau_{p}\right)^{2}\right\|^{(j-1) / 2}(2 n)^{j^{2} / 1+j-5 / 1}
\end{aligned}
$$

and $\left\|\mathcal{H}_{i}^{(2)}\right\|_{\text {ans }}$ is given in Eq. (A12), so

$$
\begin{aligned}
& \left\|\overline{\mathscr{H}}^{(j+1)}\right\|_{\mathrm{nng}} \leq F(n)^{(j-1) / 2}\left\|\left(\overline{\mathscr{K}}^{(0)} \Delta \tau_{p}\right)^{2}\right\|^{(j-1) / 2}(2 n)^{2 / 4+j-5 / 4}\left(1 / \Delta \tau_{p}\right)\left[\frac{n^{2} \alpha^{2} K^{2}}{8}\left\|\overline{\mathcal{H}}^{(0)} \Delta \tau_{p}\right\|^{2}\left\|\left(\overline{\mathcal{H}}^{(0)} \Delta \tau_{p}\right)^{2}\right\|^{2}\right. \\
& \left.+\frac{n \alpha^{3} K^{3}}{12}\left\|\left(\overline{\mathscr{K}}^{(0)} \Delta \tau_{p}\right)^{3}\right\|^{2}\right]^{1 / 2} \text {. }
\end{aligned}
$$

We define the selectivity $S$ to be $K^{1 / 2}\left\|\overline{\mathcal{H}}^{(0)}\right\| /\left\|\overline{\mathcal{F}}_{\text {nns }}^{(j+1)}\right\|$, which is the ratio of a typical nonzero matrix element of $\overline{\mathcal{K}}^{(0)}$ to a typical nonzero matrix element of $\left(\overline{\mathfrak{K}}^{(j+1)}\right)_{\mathrm{ans}}$ We would like to calculate $S$ as $\overline{\mathcal{H}}^{(0)} t_{c}$ approaches the limit of convergence, which is $\left\|\left(\overline{\mathcal{K}}^{(0)} t_{c}\right)^{2}\right\| \sim F(n)^{-1}$. Since $t_{c}=(2 n)^{(j+1) / 2} \Delta \tau_{b},\left\|\left(\overline{\mathcal{H}}^{(0)} \Delta \tau_{p}\right)^{2}\right\| \sim(2 n)^{-(j+1)} F(n)^{-1}$, and

$$
\begin{aligned}
S= & K^{1 / 2}\left\|\overline{\mathscr{K}}^{(0)}\right\| /\left\|\overline{\mathcal{K}}^{(j+1)}\right\|_{\mathrm{nms}}=K^{-1} F(n)^{1 / 2}(2 n)^{F^{2} / 4+7 / 4} \\
& \times\left[\frac{n^{2} \alpha^{2}}{8 K}+\frac{n \alpha^{3}}{12}\left(F(n)\left\|\left(\overline{\mathscr{F}}^{(0)} t_{c}\right)^{3}\right\| /\left\|\overline{\mathscr{K}}^{(0)} t_{c}\right\|\right)^{2}\right]^{1 / 2} .
\end{aligned}
$$

Equation (A15) is the same as Eq. (41).

${ }^{1}$ H. Hatanaka, T. Terao, and T. Hashi, J. Phys. Soc. Jpn. 39,835 (1975); H. Hatanaka and T. Hashi, J. Phys. Soc. Jpn. 39, 1139 (1975).

${ }^{2}$ (a) S. Vega, T. W. Shattuck, and A. Pines, Phys. Rev. Lett. 37, 43 (1976); (b) A. Pines, D. Wemmer, J. Tang, and S. Sinton, Bull. Am. Phys. Soc. 23, 21 (1978); (c) G. Drobny, A. Pines, S. Sinton, D. P. Weitekamp, and D. Wemmer, Faraday Div. Chem. Soc. Symp. 13, 49 (1979); (d) G. Bodenhausen, R. L. Vold, and R. R. Vold, J. Magn. Reson. 37, $93(1980)$

${ }^{3}$ M. E. Stoll, A. J. Vega, and R. W. Vaughan, J. Chem. Phys. 67, 2029 (1977).

${ }^{4}$ W. P. Aue, E. Bartholdi, and R. R. Ernst, J. Chem. Phys. 64, 2229 (1976); A. Wokaun and R. R. Ernst, Chem. Phys.
Lett. 52, 407 (1977); A. Wokaun and R. R. Ernst, Mol. Phys. 36, 317 (1978).

${ }^{5}$ A. Saupe, Z. Naturforsch. Teil A 20, 572 (1965).

${ }^{6}$ D. Wemmer, S. Sinton, and A. Pines (to be published); D. Wemmer, Ph. D. thesis, University of California, Berkeley, 1979 (unpublished).

${ }^{7}$ W. S. Warren, S. Sinton, D. P. Weitekamp, and A. Pines, Phys. Rev. Lett. 43, 1791 (1979).

${ }^{8} \mathrm{U}$. Haeberlen and J. S. Waugh, Phys. Rev. 175, 453 (1968).

${ }^{9}$ (a) U. Haeberlen, High Resolution NMR in Solids, Selective Averaging (Academic, New York, 1976); (b) M. Mehring, High Resolution NMR Spectroscopy in Solids (Springer, Berlin, 1976).

${ }^{10} \mathrm{~W}$. Magnus, Commun. Pure Appl. Math. 1, 649 (1954).

${ }^{11} \mathrm{~J}$. S. Waugh, L. M. Huber, and U. Haeberlen, Phys. Rev. Lett. 20, 180 (1968).

${ }^{12}$ D. P. Burum and W. K. Rhim, J. Chem. Phys. 71, 944 (1979).

${ }^{13} \mathrm{U}$. Haeberlen, Ref, 9(a), p. 178.

${ }^{14} \mathrm{~J}$. S. Waugh, "Coherent Averaging and Double Resonance in Solids," NMR-Basic Principles and Progress (Springer, Berlin, 1976), Vol. 13, p. 23-30.

${ }^{15}$ Haeberlen, Ref. 9(a), p. 98.

${ }^{16}$ W. - K. Rhim, A. Pines, and J. S. Waugh, Phys. Rev. B 3 , 684 (1971).

${ }^{17}$ W. S. Warren and A. Pines (to be published).

${ }^{18} \mathrm{This}$ condition is also satisfied for most $n k$-quantum selective sequences.

${ }^{19}$ S. Vega and A. Pines, J. Chem. Phys. 66, 5624 (1977); A. Wokaun and R. R. Ernst, J. Chem. Phys. 67, 1752 (1977); S. Vega, J. Chem. Phys. 68, 5518 (1978). 